\title{
CLASSIFICATION OF RANK-2 AMPLE AND SPANNED VECTOR BUNDLES ON SURFACES WHOSE ZERO LOCI CONSIST OF GENERAL POINTS
}

\author{
ATSUSHI NOMA
}

\begin{abstract}
Let $X$ be an $n$-dimensional smooth projective variety over an algebraically closed field $k$ of characteristic zero, and $E$ an ample and spanned vector bundle of rank $n$ on $X$. To study the geometry of $(X, E)$ in view of the zero loci of global sections of $E$, Ballico introduces a numerical invariant $s(E)$. The purposes of this paper are to give a cohomological interpretation of $s(E)$, and to classify ample and spanned rank-2 bundles $E$ on smooth complex surfaces $X$ with $s(E)=2 c_{2}(E)$, or $2 c_{2}(E)-1$; namely ample and spanned 2-bundles whose zero loci of global sections consist of general $c_{2}(E)$ points or general $c_{2}(E)-1$ points plus one. As an application of these classification, we classify rank-2 ample and spanned vector bundles $E$ on smooth complex projective surfaces with $c_{2}(E)=2$.
\end{abstract}

\section{INTRODUCTION}

Consider an $n$-dimensional smooth projective variety $X$ over an algebraically closed field $k$ of characteristic zero and a spanned vector bundle $E$ of rank $n$ on $X$ with the $n$th Chern class $c_{n}(E)$ positive. There has been a certain amount of interest recently in the problem of finding a characterization of ample and spanned vector bundles of a small top Chern class $c_{n}(E)$, in connection with the adjunction theory for ample vector bundles. To study the geometry of $(X, E)$ in view of the zero loci of global sections of $E$, Ballico [3] introduces a numerical invariant $s(E)$ as follows. If a global section $t$ is general, since $k$ is of characteristic zero, then the zero $(t)_{0}$ of $t$ consists of distinct $c:=c_{n}(E)$ points of $X$, hence, by $t \mapsto(t)_{0}$, we have a rational map $\rho$ from $H^{0}(X, E)-0 / k^{*}$ to the $c$ th symmetric product $\operatorname{Symm}^{c} X$ of $X$. Then $s(E)$ is defined by the dimension of the image of $\rho$. This invariant gives a rough indication of how spread the zero locus of a general global section of $E$ are for any embeddings, and ranges $n \leq s(E) \leq n c_{n}(E)$. In [3], it is shown that if $s(E)=n c_{n}(E)$ then $X$ is rational and that if $s(E)=n c_{n}(E)-1$ then $X$ is uniruled. And he tries to classify ample and spanned bundles $E$ on surfaces $X$ of small $c_{2}(E)$ with $s(E)=2 c_{2}(E)$ or $2 c_{2}(E)-1[3,4]$.

As his results suggest, this invariant in extremal cases seems to determine the structure of $(X, E)$ even if $c_{n}(E)$ is not small, and hence it is in fact

Received by the editors November 22, 1991 and, in revised form, September 8, 1992.

1991 Mathematics Subject Classification. Primary 14F05, 14J60.

Key words and phrases. Ample vector bundle, spanned vector bundle, zero cycle, adjunction map. 
very reasonable to expect the pair of $(X, E)$ with "extremal" $s(E)$ must be completely enumerated, at least $n=2$. As the first step to this end, one would like to have an interpretation of $s(E)$, which provides us a certain way for its calculation as well as a closer understanding of the extremal examples.

Our purposes here are to give a cohomological interpretation of this invariant and to complete Ballico's classification.

Along this line, our first result is the following:

Corollary 1.2. Let $E$ be an ample and spanned vector bundle on a smooth projective variety $X$ over an algebraically closed field $k$ of characteristic zero. If a global section $t$ of $E$ is general, then

$$
s(E)=\operatorname{dim}_{k} H^{0}(X, E)-\operatorname{dim}_{k} H^{0}\left(X, \mathscr{J}_{(t)_{0}} \otimes E\right)
$$

holds, where $\mathscr{I}_{(t)_{0}}$ denotes the ideal sheaf of the zero locus $(t)_{0}$ of $t$.

The theorem implies immediately that $s(E)-1$ is exactly equal to the dimension of the linear subspace of $\mathbf{P}\left(H^{0}(E)\right)$, spanned by the fibres $\pi^{-1}\left(P_{1}\right), \ldots$, $\pi^{-1}\left(P_{c}\right)$ of the projection $\pi: \mathbf{P}_{X}(E) \rightarrow X$ over a "general" zero locus $\left\{P_{1}, \ldots\right.$, $\left.P_{c}\right\} \quad\left(c:=c_{n}(E)\right)$. Also, this formula provides us a way for calculation of $s(E)$.

Next we classify $(X, E)$ with small $n c_{n}(E)-s(E)$. In the case $n=1$, by using (1.2), the problem reduces to classical ones (see (1.5)(1)). Hence we deal with the case of $n=\operatorname{dim} X=\operatorname{rank} E=2$.

Our results are the following:

Theorem 3.1. Let $E$ be an ample and spanned rank- 2 vector bundle on a smooth projective surface $X$ over the complex number field. Then $s(E)=2 c_{2}(E)$ if and only if $(X, E)$ is isomorphic to one of the following:

(1) $\left(\mathbf{P}^{2}, \mathscr{O}(2) \oplus \mathscr{O}(2)\right)$.

(2) $\left(\mathbf{P}^{2}, T_{\mathbf{P}}(1)\right)$, where $T_{\mathbf{P}}$ is the tangent bundle of $\mathbf{P}^{2}$.

(3) $\left(\mathbf{F}_{0}, \mathscr{O}(2 h+f) \oplus \mathscr{O}(h+2 f)\right)$.

(4) $\left(\mathbf{F}_{1}, E\right)$, where $E$ is a unique bundle defined by a nontrivial extension of $\mathscr{O}(h+3 f)$ by $\mathscr{O}(2 h+2 f)$.

(5) $\left(\mathbf{P}^{2}, \mathscr{O}(1) \oplus \mathscr{O}(1)\right)$.

(6) $\left(\mathbf{P}^{2}, \mathscr{O}(1) \oplus \mathscr{O}(2)\right)$.

(7) $\left(\mathbf{P}^{2}, T_{\mathbf{P}}\right)$.

(8) $\left(\mathbf{F}_{e}, \mathscr{O}(h+a f)^{\oplus 2}\right)(a>e \geq 0)$.

(9) $\left(\mathbf{F}_{e}, \mathscr{O}(h+a f) \oplus \mathscr{O}(h+(a+1) f)\right) \quad(a>e \geq 0)$.

Here, $\mathbf{F}_{e}$ denotes the eth Hirzebruch surface with the minimal section $h$ and a fibre $f$, and with the projection $\pi: \mathbf{F}_{e} \rightarrow \mathbf{P}^{1}$.

Theorem 4.1. Let $E$ be an ample and spanned rank- 2 vector bundle on a smooth projective surface over the complex number field. Then $s(E)=2 c_{2}(E)-1$ if and only if $(X, E)$ is isomorphic to one of the following:

(1) $\left(\mathbf{P}^{2}, \mathscr{O}(1) \oplus \mathscr{O}(3)\right)$.

(2) $\left(\mathbf{P}^{2}, F^{P}(2)\right)$, where $F^{P}$ is the vector bundle defined by an extension of the ideal sheaf $\mathscr{I}_{P}$ of $P \in \mathbf{P}^{2}$ by the structure sheaf $\mathscr{O}$ ([15, (1.33)]; see also $[4, p .484])$.

(3) $\left(\mathbf{P}^{2}, \mathscr{O}(2) \oplus \mathscr{O}(3)\right)$.

(4) $\left(\mathbf{F}_{0}, \mathscr{O}(2 h+2 f) \oplus \mathscr{O}(h+f)\right)$.

(5) $\left(\mathbf{F}_{0}, \mathscr{O}(2 h+2 f) \oplus \mathscr{O}(h+2 f)\right)$. 
(6) $\left(\mathbf{F}_{0}, \mathscr{O}(2 h+2 f) \oplus \mathscr{O}(h+3 f)\right)$.

(7) $\left(\mathbf{F}_{0}, E\right)$, where $E$ is a bundle defined by a nontrivial extension of $\mathscr{O}(h+3 f)$ by $\mathscr{O}(2 h+f)$.

(8) $\left(\mathbf{F}_{1}, \mathscr{O}(2 h+3 f) \oplus \mathscr{O}(h+2 f)\right)$.

(9) $\left(\mathbf{F}_{1}, \mathscr{O}(2 h+3 f) \oplus \mathscr{O}(h+3 f)\right)$.

(10) $\left(\mathbf{F}_{1}, E\right)$, where $E$ is a unique bundle defined by a nontrivial extension of $\mathscr{O}(h+4 f)$ by $\mathscr{O}(2 h+3 f)$.

(11) $\left(\mathbf{F}_{2}, E\right)$, where $E$ is a unique bundle defined by a nontrivial extension of $\mathscr{O}(h+4 f)$ by $\mathscr{O}(2 h+4 f)$.

(12) $\left(\mathbf{F}_{e}, \mathscr{O}(h+a f) \oplus \mathscr{O}(h+(a+2) f)\right) \quad(a>e \geq 0)$.

(13) $\left(\mathbf{P}_{C}(\mathscr{F}), \pi^{*}(\mathscr{E} \otimes \mathscr{A}) \otimes \mathscr{O}(1)\right)$, where $\mathbf{P}_{C}(\mathscr{F})$ is a geometrically ruled surface over an elliptic curve $C$ with the projection $\pi: \mathbf{P}_{C}(\mathscr{F}) \rightarrow C$ and with the tautological line bundle $\mathscr{O}(1), \mathscr{F}$ and $\mathscr{E}$ are normalized (i.e., $H^{0}(\mathscr{F}) \neq 0$ but $H^{0}(\mathscr{F} \otimes \mathcal{N})=0$ for every line bundle $\mathscr{N}$ on $C$ with $\operatorname{deg} \mathscr{N}<0$ ) rank-2 vector bundles on $C$ of $\operatorname{deg} \mathscr{F}=-e$ and $\operatorname{deg} \mathscr{E}=1$, and $\mathscr{A}$ is a line bundle on $C$ of $\operatorname{deg} \mathscr{A}=\alpha$ with $\alpha-e \geq 1$.

The theorems above complete Ballico's classification of rank-2 ample and spanned vector bundles with $2 c_{2}(E)-s(E)=0$ and $2 \leq c_{2}(E) \leq 7$ or, $2 c_{2}(E)-s(E)=1$ and $3 \leq c_{2}(E) \leq 7$ in [4], where he assumes, in addition, that $\operatorname{det}(E)$ is very ample. Unfortunately, Ballico's classification in the case $s(E)=2 c_{2}(E)-1$ contains two omissions; the bundles with the ample adjoint bundles $K \otimes \operatorname{det}(E)$ and with $h^{0}(K \otimes \operatorname{det}(E))=c_{2}(E)$, and the bundles on elliptic geometrically ruled surfaces corresponding to (13) in Theorem 4.1 are omitted. The bundles of the first type were pointed out by Matsubara, and are classified in [24]. The omission of the second type results from his incorrect assertion $[4,(1.1)]$, which ruled out the possibility of ruled surfaces of the first irregularity greater than or equal to 1 : Precisely, in the proof $[4, p$. 473, line 7], the elliptic curves have been excluded from projective curves with infinitely many involutions by mistake. Due to these two faults, his preliminary lemmas in $[4, \S 1]$ are not true. Here we will restate and prove some of these assertions (see §4). The ideas of our proof are found in Ballico [4, §1]. In our proof, we clarify the meaning of "a general point in a space" by constructing the corresponding open subset actually, though this makes our proof somewhat longer.

Theorems 3.1 and 4.1, together with Ballico's classification of ample and spanned rank-2 bundles on surfaces with the other extremal $s(E)[3,(4.1)]$, yield the following theorem.

Theorem 6.1. Let $E$ be an ample and spanned rank-2 vector bundle on a smooth projective surface $X$ over the complex number field. Then $c_{2}(E)=2$ if and only if $(X, E)$ is one of the following:

(1) $(X, E) \cong\left(\mathbf{P}^{2}, \mathscr{O}(1) \oplus \mathscr{O}(2)\right)$.

(2) $(X, E) \cong\left(\mathbf{Q}^{2}, \mathscr{O}(1)^{\oplus 2}\right)$, where $\mathbf{Q}^{2}$ is a smooth quadric in $\mathbf{P}^{3}$.

(3) $X$ is isomorphic to a geometrically ruled surface $\mathbf{P}_{C}(\mathscr{F})$ over an elliptic curve $C$ with the projection $\pi: \mathbf{P}_{C}(\mathscr{F}) \rightarrow C$ and with the tautological line bundle $\mathscr{O}(1)$, and $E \cong \pi^{*}(\mathscr{E}) \otimes \mathscr{O}(1)$. Here $\mathscr{F}$ and $\mathscr{E}$ are indecomposable rank-2 vector bundles on $C$ of degree 1 .

(4) There exists a finite morphism $f: X \rightarrow \mathbf{P}^{2}$ of degree 2 and $E \cong$ $f^{*}\left(\mathscr{O}(1)^{\oplus 2}\right)$. 
The problem of classifying ample and spanned rank-2 vector bundles $E$ of $c_{2}(E)=2$ on smooth complex projective surfaces $X$ was already considered by many authors: Lanteri [20, 21] and Ballico and Lanteri [6] classified these bundles under the assumption that $\operatorname{det}(E)$ are very ample; Biancofiore, Fania, and Lanteri [8] and Ballico and Lanteri [7] classified these bundles in the full situation. However, H. Maeda told me that the papers $[20,8]$ contain the same gaps, for which I cannot give a proof: Namely for $(X, E)$, the morphism $\mu: X \rightarrow \operatorname{Grass}\left(2, H^{0}(X, E)\right)$ sending $x \in X$ to a 2-quotient $H^{0}(X, E) \rightarrow$ $E \otimes k(x)$ factors through the adjunction mapping of $\operatorname{det}(E)$. Consequently, the classification seems to have been incomplete, since the proof of the classification theorems in [6] and [7] rely on the results of [8] and [20]. Here we prove the theorem from a different point of view. Hence, apart from the above point, we believe that our method is worth dealing with.

Our exposition proceeds as follows. In the first section, we give an interpretation of Ballico's invariant $s(E)$. Here we define an invariant $s p(E)$ for a vector bundle of rank $r(\leq n)$ on a projective variety of dimension $n$, by using a morphism $\tau$ from an open set of global sections to a Hilbert scheme. This invariant is equal to $s(E)$ when $r=n$ and $X$ is smooth over a field of characteristic zero. The main results (1.1) and (1.2), an interpretation of $s p(E)$ and $s(E)$, are obtained by looking at the fibre of the morphism $\tau$. Moreover, we look at the map between tangent spaces of $\tau$.

The following four sections are devoted to the classification of rank-2 vector bundles $E$ over smooth complex projective surfaces $X$ with $2 c_{2}(E)-s(E)=$ 0 or 1 . In $\S 2$, as preliminaries of the classification, we prove some lemmas useful for our classification, and recall some results. The classifications in the case

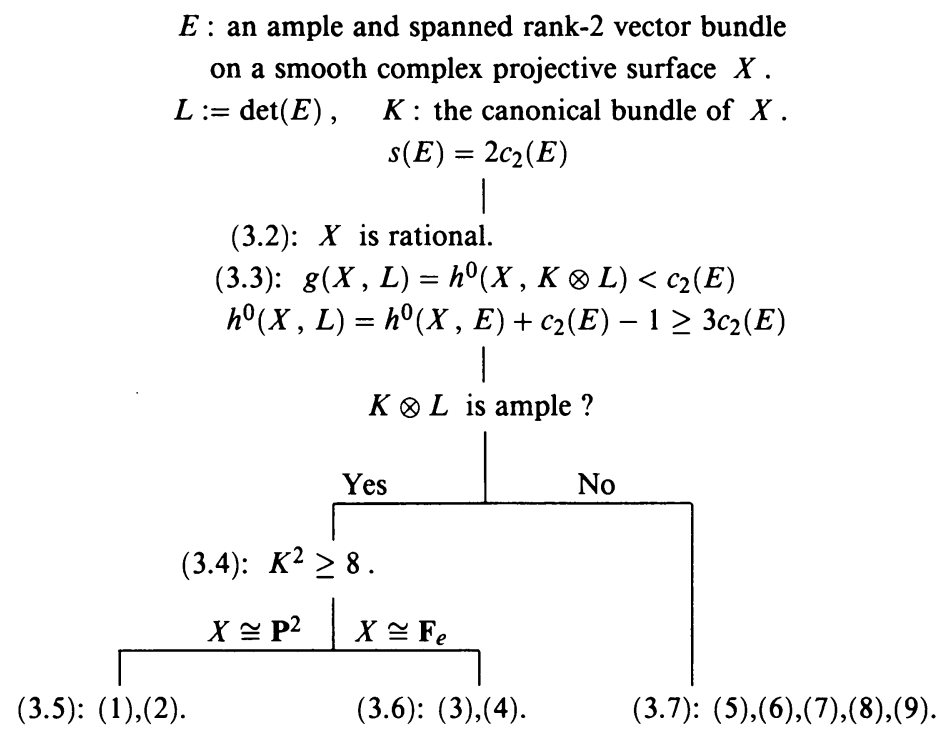

Figure 1. Classification in case $s(E)=2 c_{2}(E)$ (Theorem 3.1) 


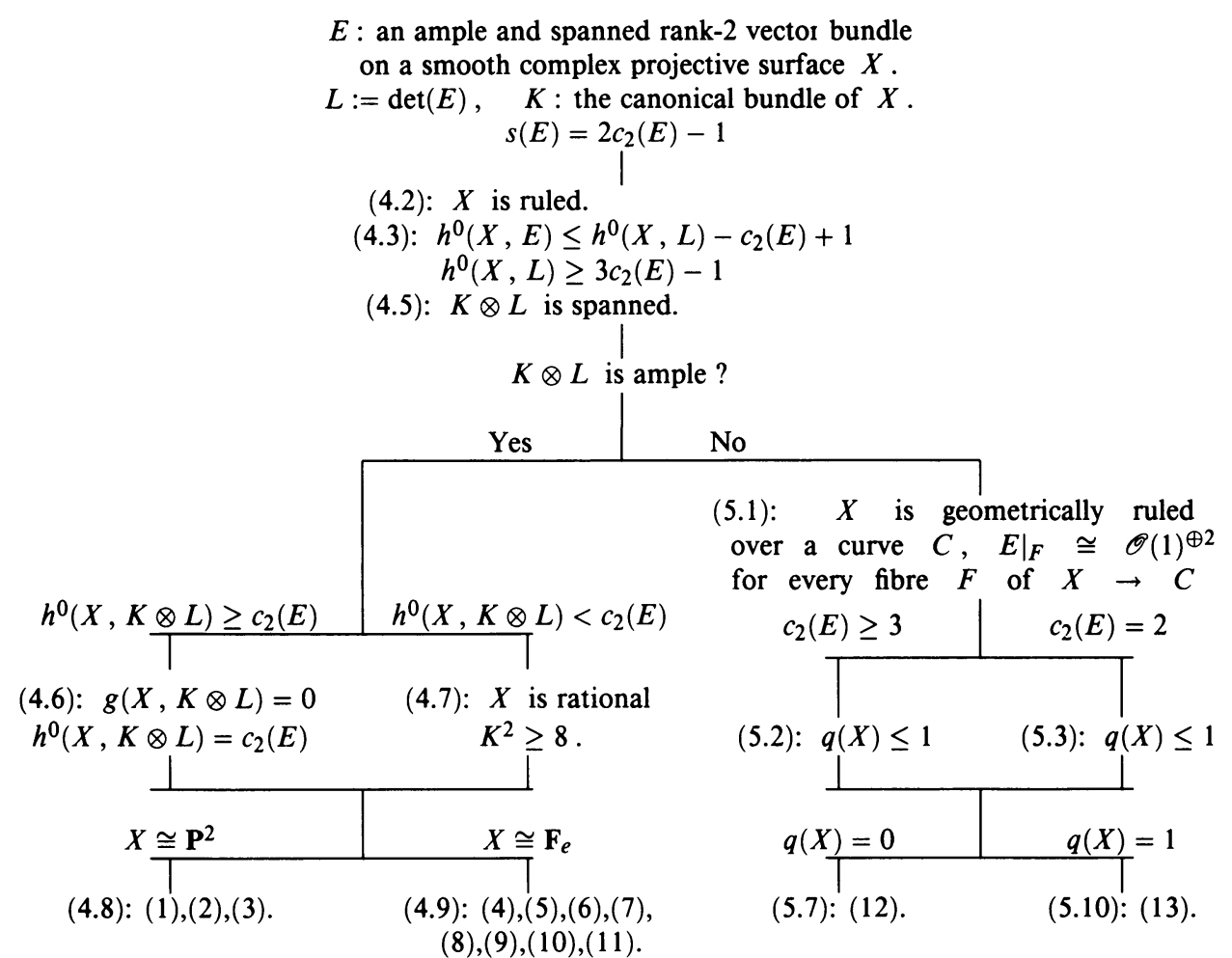

Figure 2. Classification in case $s(E)=2 c_{2}(E)-1$ (Theorem 4.1)

$2 c_{2}(E)-s(E)=0$ and 1 are carried out in $\S 3$ and $\S 4,5$, respectively. The classifications in the both cases proceed in a similar way (for details, see the charts of classifications (Figures 1 and 2)): Based on the adjunction theory for ample vector bundles, our proof of classification theorems consists of two parts. In the first part, we treat the bundles $E$ with the ample adjoint bundles $K \otimes \operatorname{det}(E)$. The key step to this part is to deduce that an underlying surface is the projective plane or a Hirzebruch surface. To determine the bundles, the inequalities in [ 3 , 4] (see (3.3) and (4.3)), going back to Griffiths and Harris [15], are used. In the second part, we treat the bundles $E$ with the not ample adjoint bundles. By a result of Fujita [11] (see (2.4)), the structures of $(X, E)$ are roughly described. At this stage, the formula in (1.2) is a powerful tool for the determination of the structure of $(X, E)$ precisely. But, in case $s(E)=2 c_{2}(E)-1$, we need several steps further, so the classification in this case are carried out in $\S 5$. In the course of the determination of bundles on an elliptic geometrically ruled surface with $s(E)=2 c_{2}(E)-1$, we encounter ample and spanned rank-2 bundles with $s(E)=2 c_{2}(E)-2$. These bundles are counterexamples to (6.1) in [4] and to $(0.1)$ in [5] (see (5.11)).

In the final section $(\S 6)$, as an application of $\S 3,4$, and 5 , we classify the rank-2 ample and spanned vector bundles of $c_{2}(E)=2$ on smooth projective surfaces $(6.1)$.

I am grateful to Professors Takashi Matsubara, Hajime Kaji, Toshio Hosoh, 
and Hidetoshi Maeda for valuable discussions and kind advice during the preparation of this paper. I am especially indebted to the referee for suggesting the simple argument of the proof of (1.1).

\section{A COHOMOLOGICAL INTERPRETATION OF BALLICO'S INVARIANT $s(E)$}

Let $X \subseteq \mathbf{P}^{m}$ be a Cohen-Macaulay projective variety of dimension $n$ over an algebraically closed field $k$. Let $E$ be a vector bundle of rank $r(\leq n)$ on $X$. We set $|E|=\mathbf{P}_{k}\left(H^{0}(E)^{\vee}\right)=H^{0}(E)-0 / k^{*}$. A closed point of $|E|$ represented by $t \in H^{0}(E)$ is denoted by $[t]$. For a global section $t$, we denote the zero locus of $t$ by $(t)_{0}$. Recall that $(t)_{0}$ is locally defined as follows: Let $\psi^{U}: E \mid U \rightarrow \mathscr{O}_{U}^{\oplus r}$ be a local trivialization over an affine open subset $U$ of $X$ and $\psi_{i}^{U}: E \mid U \rightarrow \mathscr{O}_{U}$ the composition of $\psi^{U}$ and the $i$ th projection. Then $(t)_{0}$ is defined over $U$ by the ideal generated by $\psi_{1}^{U}(t), \ldots, \psi_{r}^{U}(t)$ (see [13, p. 430 B.3.]). In other words, $(t)_{0}=\operatorname{Spec}_{X}\left(\operatorname{Coker}\left(t^{\vee}: E^{\vee} \rightarrow \mathscr{O}_{X}\right)\right.$ ) (see [18, p. 128, Example 5.17]). Next we recall the construction of the scheme-theoretic family $\mathscr{Z}$ of zeros of global sections parametrazed by $|E|$. Let $\mathscr{D}_{E}$ be the cokernel of the dual $\mathrm{ev}^{\vee}: E^{\vee} \rightarrow H^{0}(E)^{\vee} \otimes \mathscr{O}_{X}$ of the evaluation map of $E$. So $\mathscr{Z}:=\mathbf{P}_{X}\left(\mathscr{D}_{E}\right)$ is a subscheme of $|E| \times X=\mathbf{P}_{X}\left(H^{0}(E)^{\vee} \otimes \mathscr{O}_{X}\right)$ with the projection $\Phi: \mathscr{Z} \rightarrow|E|$. Then a fibre of $\Phi$ over $[t]$ is $\operatorname{Spec}(k) \times|E| \mathcal{Z}=$ $\mathbf{P}_{X}\left(\mathscr{O}_{X}\right) \times_{|E| \times X} \mathbf{P}_{X}\left(\mathscr{D}_{E}\right)=\mathbf{P}_{X}\left(\mathscr{O}_{X} /\left(t^{\vee} \otimes 1_{\mathscr{O}_{X}}\right)\left(\operatorname{ev}^{\vee}\left(E^{\vee}\right)\right)\right) \quad($ see [16, II, 2.5.13]), hence $\Phi^{-1}([t])=(t)_{0}$, since $\operatorname{Coker}\left(t^{\vee}: E^{\vee} \rightarrow \mathscr{O}_{X}\right) \cong \mathscr{O}_{X} /\left(t^{\vee} \otimes 1_{\mathscr{O}_{X}}\right)\left(\operatorname{ev}^{\vee}\left(E^{\vee}\right)\right)$. More generally, let $\mathscr{E}$ be a locally free sheaf on a projective scheme $\mathscr{Z}$ flat over a Noetherian $k$-algebra $R$ and $s$ a global section such that $s: R \rightarrow H^{0}(\mathscr{E})$ is a split injection. In this case, the zero $(s)_{0}$ and the family of zeros are defined by the same way. Therefore, the base change $\operatorname{Spec}(R) \times_{|E|} \mathscr{Z}$ by a $k$-morphism $f: \operatorname{Spec}(R) \rightarrow|E|$ is the zero of the global section, corresponding to the $R$ valued point $(f, 1): \operatorname{Spec}(R) \rightarrow\left|E \otimes_{k} R\right|=|E| \times \operatorname{Spec}(R)$, of the bundle $E \otimes_{k} R$ on $X \times \operatorname{Spec}(R)$.

From now on towards the end of this section, we always assume furthermore that $E$ is globally generated with $h^{0}(X, E)=N+1$ and that there exists a nonzero global section $t$ with $\operatorname{dim}(t)_{0}=n-r$. The last assumption holds whenever $E$ is an ample and spanned vector bundle on a smooth projective variety over a field $k$ of characteristic zero by $[9,(2.5)]$. Set $P(z)$ be the Hilbert polynomial with respect to $(t)_{0} \quad(\subset X) \subseteq \mathbf{P}^{m}$. Set $|E|_{\text {reg }}=\left\{x \in|E| ; \operatorname{dim} \Phi^{-1}(x)=n-r\right\}$. By Chevalley's theorem [16, IV, 13.1.5], $|E|_{\text {reg }}$ is a nonempty open subset of $|E|$, since $\operatorname{dim} \mathscr{Z}=N+n-r$ and since the dimension of general fibre of $\Phi$ is less than or equal to $n-r$ by assumption. We say that a global section $t \in H^{0}(E)$ is regular if $[t] \in|E|_{\text {reg }}$, i.e., if $\operatorname{dim}(t)_{0}=n-r$. Since $\mathscr{Z}$ is $\mathbf{P}^{(N-r)}$-bundle over $X$ and hence $\mathscr{Z}$ is Cohen-Macaulay, $\left.\Phi\left|\Phi^{-1}\left(|E|_{\text {reg }}\right): \Phi^{-1}\left(|E|_{\text {reg }}\right) \rightarrow\right| E\right|_{\text {reg }}$ is a flat morphism. Thus, by the representability of the Hilbert scheme, there exists a morphism of schemes $\tau:|E|_{\mathrm{reg}} \rightarrow \mathrm{Hilb}_{X / k}^{P(z)}$. Here $\mathrm{Hilb}_{X / k}^{P(z)}$ is the Hilbert scheme representing the contravariant functor $\mathscr{H} i l b_{X / k}^{P(z)}$, from the category of locally noetherian $k$-schemes $T$ to the set $\mathscr{H} i l b_{X / k}^{P(z)}(T)$ of closed subschemes $M$ of $X \times T$ flat over $T$ with each fibre of the Hilbert polynomial $P(z)$ (see [17]).

Then we define the invariant $s p(E)$ by

$$
s p(E)=\operatorname{dim} \tau\left(|E|_{\mathrm{reg}}\right) .
$$


This invariant $s p(E)$ is interpreted as follows:

Proposition 1.1. Let $E$ be a globally generated vector bundle of rank $r$ on $a$ Cohen-Macaulay projective variety $X$ of dimension $n(\geq r)$. Assume that there exists a regular global section. Then for a regular section $t \in H^{0}(E)$, the fibre of $\tau:|E|_{\mathrm{reg}} \rightarrow \mathrm{Hilb}_{X / k}^{P(z)}$ over $\tau([t])$ is just $\left|\mathscr{J}_{(t)_{0}} \otimes E\right| \cap|E|_{\mathrm{reg}}$, where $\mathscr{J}_{(t)_{0}}$ denotes the ideal sheaf of the zero locus of $t$ and $\left|\mathscr{I}_{(t)_{0}} \otimes E\right|=\mathbf{P}\left(H^{0}\left(\mathscr{I}_{(t)_{0}} \otimes E\right)^{\vee}\right)=$ $H^{0}\left(\mathscr{I}_{(t)_{0}} \otimes E\right)-0 / k^{*} \subset|E|$. Thus $s p(E)=h^{0}(E)-h^{0}\left(\mathscr{I}_{(t)_{0}} \otimes E\right)$ holds for $a$ general regular global section $t \in H^{0}(E)$.

Proof. We consider the short exact sequence $0 \rightarrow H^{0}\left(\mathscr{J}_{(t)_{0}} \otimes E\right) \rightarrow H^{0}(E) \rightarrow$ $H^{0}\left(\mathscr{O}_{(t)_{0}} \otimes E\right)$. If a global section $t^{\prime}$ satisfies $\left[t^{\prime}\right] \in\left|\mathscr{I}_{(t)_{0}} \otimes E\right| \cap|E|_{\text {reg }}$, then $t^{\prime}=0$ in $H^{0}\left(\mathscr{O}_{(t)_{0}} \otimes E\right)$. Thus for every affine open subset $U$ where $E$ is trivialized as $\psi^{U}: E \mid U \rightarrow \mathscr{O}_{U}^{\oplus r}, t_{i}^{\prime}:=\psi_{i}^{U}\left(t^{\prime}\right)(i=1, \ldots, r)$ are contained in $\mathscr{F}_{(t)_{0}} \mid U \subseteq \mathscr{O}_{U}$. In other words, $\left(t^{\prime}\right)_{0} \supseteq(t)_{0}$, and hence $\left(t^{\prime}\right)_{0}=(t)_{0}$, since $\left(t^{\prime}\right)_{0}$ and $(t)_{0}$ belong to the same flat family. Conversely, if $\left(t^{\prime}\right)_{0}=(t)_{0}$ as schemes, then $t^{\prime} \mid U=0 \in H^{0}\left(U, \mathscr{O}_{(t)_{0}} \otimes E \mid U\right)$ for every $U$ where $E \mid U$ is trivial. Thus $t^{\prime}=0$ in $H^{0}\left(\mathscr{O}_{(t)_{0}} \otimes E\right)$ and hence $\left[t^{\prime}\right] \in\left|\mathscr{I}_{(t)_{0}} \otimes E\right| \cap|E|_{\text {reg }}$, as required.

Now we recall the invariant $s(E)$ of Ballico [3] (see also [4, 5]). Let $E$ be a globally generated vector bundle of rank $r=n$ with $c_{n}(E)>0$ on a smooth projective variety $X$ of dimension $n$ over an algebraically closed field $k$ of characteristic 0 . Then regular global sections actually exist. Since for a regular global section $t \in H^{0}(E),(t)_{0}$ is 0 -dimensional of $\operatorname{deg}(t)_{0} \quad\left(:=\right.$ length $\left.\mathscr{O}_{(t)_{0}}\right)=$ $c_{n}(E)$ (see [18, p. 431]), we have a map $\rho:|E|_{\text {reg }} \rightarrow \operatorname{Symm}_{k}^{c}(X)$ by $[t] \mapsto(t)_{0}$, where $\operatorname{Symm}_{k}^{c}(X)$ is the $c:=c_{n}(E)$ th symmetric product of $X$. One checks easily that $\rho$ is a morphism of schemes. Then the invariant $s(E)$ is defined by

$$
s(E)=\operatorname{dim} \rho\left(|E|_{\mathrm{reg}}\right) .
$$

To see the relation between $s p(E)$ and $s(E)$, we consider the norm morphism $n_{X / k}: \operatorname{Hilb}_{X / k}^{c_{n}(E)} \rightarrow \operatorname{Symm}_{k}^{c}(X)$ (for more details, see [17, p. 25]). Then the morphism $\rho$ is nothing but $n_{X / k} \circ \tau$. Since $n_{X / k}$ is isomorphic over the open subset parametrizing smooth distinct $c_{n}(E)$-point of $X$, by the generic smoothness of $\Phi$, we have $\operatorname{dim} \rho\left(|E|_{\text {reg }}\right)=\operatorname{dim} \tau\left(|E|_{\text {reg }}\right)$. Thus we have

Corollary 1.2. Let $E$ be a globally generated vector bundle of rank $n$ with $c_{n}(E)>0$ on a smooth projective variety $X$ of dimension $n$ over an algebraically closed field $k$ of characteristic zero. Then $s(E)=h^{0}(E)-h^{0}\left(\mathscr{J}_{(t)_{0}} \otimes E\right)$ holds for a general regular global section $t \in H^{0}(E)$.

We say that a global section $t \in H^{0}(E)$ computes $s(E)$ if $s(E)=h^{0}(E)-$ $h^{0}\left(\mathscr{I}_{(t)_{0}} \otimes E\right)$ holds.

Returning to our original situation, we shall look at the linear maps between tangent spaces of $\tau:|E|_{\mathrm{reg}} \rightarrow \mathrm{Hilb}_{X / k}^{P(z)}$. Fix a regular global section $t \in H^{0}(E)$. To this end, for the natural projection $\pi: H^{0}(E)-0 \rightarrow|E|$, we look at the composition $T_{\pi} \circ T_{\tau}: T_{t} H^{0}(E) \rightarrow T_{[t]}|E| \rightarrow T_{(t)_{0}} \mathrm{Hilb}_{X / k}^{P(z)}$. Here note that the map $T_{\pi}: T_{t} H^{0}(E) \rightarrow T_{[t]}|E|$ of tangent spaces at $t$ is the quotient $H^{0}(E) \rightarrow$ $H^{0}(E) / t \cdot k$. In general, tangent vectors are interpreted as follows: Let $\Lambda$ be the $k$-algebra $k[\epsilon] /\left(\epsilon^{2}\right), \eta: k[\epsilon] /\left(\epsilon^{2}\right) \rightarrow k$ the homomorphism with $\eta(\epsilon)=0$, 
and $l: \operatorname{Spec}(k) \hookrightarrow \operatorname{Spec}(\Lambda)$ the inclusion associated with $\eta$. If $M$ be a variety, then the tangent vectors $v \in \operatorname{Hom}_{\mathscr{O}_{M, x}}\left(\Omega_{M, x}, k\right)=\operatorname{Hom}_{k}\left(m_{x} / m_{x}^{2}, k\right)$ are in one-to-one correspondence with $k$-morphisms $\varphi: \operatorname{Spec}(\Lambda) \rightarrow M$, associating to the ring homomorphism $\mathscr{O}_{x, M} \rightarrow \Lambda, a \mapsto a+v\left(d_{M / k}(a)\right) \epsilon$, with $\varphi \circ l$ the point $\{x\}$. Here $m_{x}$ is the maximal ideal of $\mathscr{O}_{M, x}$ and $d_{M / k}$ is the differential of $M$ over $k$. Under this interpretation, the map $T_{\tau}: T_{[t]}|E| \rightarrow$ $T_{(t)_{0}} \operatorname{Hilb}_{X / k}^{P(z)}$ sends the tangent vector $v: \operatorname{Spec}(\Lambda) \rightarrow|E|$ at $[t]$ to $\tau \circ v$. By the representability of $\operatorname{Hilb}_{X / k}^{P(z)}$, the tangent vectors $w: \operatorname{Spec}(\Lambda) \rightarrow \operatorname{Hilb}_{X / k}^{P(z)}$ to $(t)_{0}$ are in correspondence with the first-order deformations of $(t)_{0} \subset X$. Moreover, the first-order deformations can be seen the global section of the normal sheaf $\mathscr{N}_{(t)_{0} / X}=\mathscr{O}_{(t)_{0}} \otimes E$ (see, for example, [2, (6.1); 17; 18, p. 267, Example 9.7]). Thus the linear map $T_{\tau} \circ T_{\pi}: T_{t} H^{0}(E) \rightarrow T_{(t)_{0}} \mathrm{Hilb}_{X / k}^{P(z)}$ of tangent spaces is a linear map $H^{0}(E) \rightarrow H^{0}\left(\mathscr{O}_{(t)_{0}} \otimes E\right)$. Then we claim that $T_{\tau} \circ T_{\pi}: H^{0}(E) \rightarrow$ $H^{0}\left(\mathscr{O}_{(t)_{0}} \otimes E\right)$ is just the restriction map. Indeed, a tangent vector $u \in H^{0}(E)$ to $H^{0}(E)$ at $t$ are sent to a tangent vector $\operatorname{Spec}(\Lambda) \rightarrow|E|$ which associates the ring homomorphism $\operatorname{Symm}\left(H^{0}(E)^{\vee}\right) \rightarrow \Lambda, g \in H^{0}(E)^{\vee} \mapsto t(g)+u(g) \epsilon$, and to the first order deformation $\operatorname{Spec}(\Lambda) \times_{|E|} \mathscr{Z} \subset \operatorname{Spec}(\Lambda) \times X$ which is the zero of the global section $t+u \epsilon$ of vector bundle $E \otimes \Lambda$ on $X \times \operatorname{Spec}(\Lambda)$ defined locally by the ideal $\left(\psi_{1}^{U}(t)+\psi_{1}^{U}(u) \epsilon, \ldots, \psi_{r}^{U}(t)+\psi_{r}^{U}(u) \epsilon\right) \subseteq \mathscr{O}_{U} \otimes \Lambda$, where $U$ is affine open over which $E$ is trivialized by $\psi^{U}: E \mid U \rightarrow \mathscr{O}_{U}^{\oplus r}$. Under the isomorphism of the set of first-order deformations of $(t)_{0} \subset X$ to $H^{0}\left(\mathscr{O}_{(t)_{0}} \otimes E\right)$, $(t+u \epsilon)_{0}$ corresponds to $u \mid(t)_{0} \in H^{0}\left(\mathscr{O}_{\left(t_{0}\right.} \otimes E\right)$, as required.

Thus we have

Proposition 1.3. The morphism $\tau:|E|_{\mathrm{reg}} \rightarrow \mathrm{Hilb}_{X / k}^{P(z)}$ induces a separable morphism of $|E|_{\mathrm{reg}}$ onto the closure of its image $\tau\left(|E|_{\mathrm{reg}}\right)$.

Remark 1.4. The argument of the proof of (1.1) and hence (1.2) is due to referee. In our original proof, to see the dimension of image of $\tau\left(|E|_{\mathrm{reg}}\right)$, we calculated the rank of the linear map of tangent spaces of $\tau$ by looking at its kernel as in (1.3).

Examples 1.5. (1). Let $L$ be an ample and spanned line bundle on a smooth projective curve $C$ over an algebraically closed field $k$ of characteristic zero. Then $s(L)=h^{0}(C, L)-1$. By using Riemann-Roch and Clifford's Theorem, we have

(a) $s(L)=c_{1}(L)$ if and only if $(C, L)$ is isomorphic to $\left(\mathbf{P}^{1}, \mathscr{O}_{\mathbf{P}}(l)\right)$ for some $l>0$.

(b) $s(L)=c_{1}(L)-1$ if and only if one of the following:

(i) $C$ is an elliptic curve and $c_{1}(L) \geq 2$.

(ii) $C$ is a hyperelliptic curve of genus $g \geq 2$ and $|L|=g_{2}^{1}$.

(c) $s(L)=c_{1}(L)-2$ if and only if one of the following:

(i) $C$ is of genus 2 , and $c_{1}(L) \geq 4$ or $|L|$ is a base-point-free $g_{3}^{1}$.

(ii) $C$ is of genus $g \geq 3$, and $|L|$ is a base-point-free $g_{3}^{1}$ or $g_{4}^{2}$.

(2) Let $T_{\mathbf{P}}$ be the tangent bundle of the projective $n$-space $\mathbf{P}^{n}$. Then, by using the Koszul complex for $T_{\mathbf{P}}$ (see, for example, [13, p. 431]) and Bott 
formula (see, for example, [28, p. 8]), we have

$$
s\left(T_{\mathbf{P}}(a)\right)= \begin{cases}n, & \text { if } a=-1, \\
n(n+1), & \text { if } a=0, \\
\left(\begin{array}{l}
a+n \\
a+1
\end{array}\right)(n+a+2)-1, & \text { if } a>0 .\end{cases}
$$

\section{Preliminaries for Classification}

In the following three sections, we classify ample and spanned rank-2 vector bundles on smooth projective surfaces with $2 c_{2}(E)-s(E)=0,1$.

From now on we work over the complex number field $\mathbb{C}$ and use the following notation and terminology. By $K_{V}$ (or $K$ ) we denote the canonical bundle of a smooth projective variety $V$ of dimension $n$. By $q(V)$, we denote the first irregularity $h^{1}\left(V, \mathscr{O}_{V}\right)$. For an ample line bundle $\mathscr{L}$ on $V$, the $\Delta$-genus $\Delta(V, \mathscr{L})$ of $(V, \mathscr{L})$ is defined by $\Delta(V, \mathscr{L})=n+\mathscr{L}^{n}-h^{0}(V, \mathscr{L})$, and the sectional genus $g(V, \mathscr{L})$ of $(V, \mathscr{L})$ by $2 g(V, \mathscr{L})-2=\left(K_{V}+(n-\right.$ 1) $\mathscr{L}) \cdot \mathscr{L}^{n-1}$ (for details, see [10]). For a line bundle $\mathscr{M}$ on $V,|\mathscr{M}|$ denotes the complete linear system associated with $\mathscr{M}$. If $\mathscr{M}$ is spanned, $\phi_{|\mathscr{K}|}: V \rightarrow$ $\mathbf{P}\left(H^{0}(V, \mathscr{M})\right)$ denotes the morphism associated with the complete linear system $|\mathscr{M}|$. We say that a short exact sequence $0 \rightarrow \mathscr{F}^{\prime} \rightarrow \mathscr{F} \rightarrow \mathscr{F} \prime \prime \rightarrow 0$ of sheaves on $V$ is globally exact if this sequence induces an exact sequence $0 \rightarrow$ $H^{0}\left(\mathscr{F}^{\prime}\right) \rightarrow H^{0}(\mathscr{F}) \rightarrow H^{0}(\mathscr{F} \prime \prime) \rightarrow 0$. A vector bundle $\mathscr{E}$ on a smooth complete curve is said to be normalized if $H^{0}(\mathscr{E}) \neq 0$ but $H^{0}(\mathscr{E} \otimes \mathscr{N})=0$ for every line bundle $\mathscr{N}$ on $C$ with $\operatorname{deg} \mathscr{N}<0$.

In this section, we provide some useful lemmas and results for our classification. The first lemma, especially (4), is a key to the case of $K \otimes L$ ample.

Lemma 2.1. Let $E$ be an ample and spanned rank-2 vector bundle on a smooth projective surface $X$. Set $L=\operatorname{det}(E), \delta=2 c_{2}(E)-s(E) \geq 0$, and $m=$ $h^{0}(E)-s(E)-1 \geq 0$. Let $\mathbf{P}_{X}(E)$ be the projective space bundle associated with $E$, with the projection $\pi: \mathbf{P}_{X}(E) \rightarrow X$, and with the tautological line bundle $\mathscr{O}(1)$. Then:

(1) $g(X, L)=h^{0}(L \otimes K)+q(X)-p_{g}(X)$;

(2) $g(X, L)=g\left(\mathbf{P}_{X}(E), \mathscr{O}(1)\right)$;

(3) $d\left(\mathbf{P}_{X}(E), \mathscr{O}(1)\right)=\Delta\left(\mathbf{P}_{X}(E), \mathscr{O}(1)\right)-2+2 c_{2}(E)-\delta+m$;

(4) $\Delta\left(\mathbf{P}_{X}(E), \mathscr{O}(1)\right)+g(X, L \otimes K)=K^{2}+3\left\{h^{0}(L \otimes K)-c_{2}(E)\right\}+3 q(X)-$ $3 p_{g}(X)+\delta-m$.

Proof. (1) This follows from an exact sequence $\left.0 \rightarrow K \rightarrow L \otimes K \rightarrow L \otimes K\right|_{A} \rightarrow 0$ for an irreducible and reduced member $A$ of $|L|$, by using Kodaira vanishing and the adjunction formula.

(2) Let $H$ be a smooth irreducible member of $|\mathscr{O}(1)|$ and $\varphi$ the birational morphism from $H$ to $X$, hence $\varphi^{*}(K)-K_{H}$ is a union of exceptional divisors for $\varphi$. Set $\mathscr{O}_{H}(1)=\mathscr{O}(1) \otimes \mathscr{O}_{H}$. By definition, $g\left(\mathbf{P}_{X}(E), \mathscr{O}(1)\right)=$ $g\left(H, \mathscr{O}_{H}(1)\right)$. Since $K_{H}=\left.\left(K_{\mathrm{P}} \otimes \mathscr{O}(1)\right)\right|_{H}=\mathscr{O}_{H}(-1) \otimes \varphi^{*}(L \otimes K)$, we have

$$
\begin{aligned}
2 g\left(H, \mathscr{O}_{H}(1)\right)-2 & =\left(\mathscr{O}_{H}(1) \otimes K_{H}\right) \cdot \mathscr{O}_{H}(1) \\
& =\varphi^{*}(L \otimes K) \cdot\left(\varphi^{*}(L)+\varphi^{*}(K)-K_{H}\right) \\
& =(L \otimes K) \cdot L=2 g(X, L)-2 .
\end{aligned}
$$


Thus $g\left(\mathbf{P}_{X}(E), \mathscr{O}(1)\right)=g\left(H, \mathscr{O}_{H}(1)\right)=g(X, L)$. For another proof, see [12, (1.2)].

(3) This follows immediately from the definition of $\Delta$-genus.

(4) By the relation between total Chern class and total Segre class in the case of $\operatorname{dim} X=\operatorname{rank} E=2, d\left(\mathbf{P}_{X}(E), \mathscr{O}(1)\right)=H^{3}=c_{1}(E)^{2}-c_{2}(E)$ (see [10, p. $12(3.7) ; 13])$. Hence, by (3), we have $\Delta\left(\mathbf{P}_{X}(E), \mathscr{O}(1)\right)=c_{1}(E)^{2}-3 c_{2}(E)+2+$ $\delta-m$. On the other hand, by the adjunction formula and (1),

$$
\begin{aligned}
g(X, L \otimes K) & =K^{2}+3 g(X, L)-c_{1}(E)^{2}-2 \\
& =K^{2}+3 h^{0}(X, L \otimes K)+3 q(X)-3 p_{g}(X)-c_{1}(E)^{2}-2 .
\end{aligned}
$$

Therefore, we have

$$
\begin{aligned}
& \Delta\left(\mathbf{P}_{X}(E), \mathscr{O}(1)\right)+g(X, L \otimes K) \\
& \quad=K^{2}+3\left\{h^{0}(L \otimes K)-c_{2}(E)\right\}+3 q(X)-3 p_{g}(X)+\delta-m .
\end{aligned}
$$

The following lemma is useful for calculation of $s(E)$.

Lemma 2.2. Let $E$ be a rank-2 ample and spanned vector bundle on a smooth projective surface $X$. Then:

(1) Assume that $h^{1}\left(E^{\vee}\right)=0$. Then $s(E)=h^{0}(E)-h^{0}\left(E \otimes E^{\vee}\right)$.

(2) Assume that $E$ is a direct sum of line bundles $M_{1}$ and $M_{2}$ on $X$. Let $D_{i}$ be a smooth irreducible member of $\left|M_{i}\right|(i=1,2)$. Then:

(2a) $s(E)=h^{0}\left(M_{1}\right)+h^{0}\left(M_{2}\right)-h^{0}\left(M_{1}^{-1} \otimes M_{2}\right)-h^{0}\left(M_{1} \otimes M_{2}^{-1}\right)-2$.

(2b) $2 c_{2}(E)-s(E) \geq \Delta\left(D_{1},\left.M_{2}\right|_{D_{1}}\right)+\Delta\left(D_{2},\left.M_{1}\right|_{D_{2}}\right) \geq 0$.

Proof. Consider the Koszul sequence for a section $t$ computing $s(E)$

$$
0 \longrightarrow \operatorname{det}\left(E^{\vee}\right) \longrightarrow E^{\vee} \longrightarrow \mathscr{I}_{(t)_{0}} \longrightarrow 0 .
$$

Tensoring this by $E$, we have

$$
0 \longrightarrow E^{\vee} \longrightarrow E^{\vee} \otimes E \longrightarrow \mathscr{I}_{(t)_{0}} \otimes E \longrightarrow 0 .
$$

(1) By assumption and the vanishing theorem [23, (1.7)], $h^{i}\left(E^{\vee}\right)=0 \quad(i=$ $0,1)$, so we have $h^{0}\left(\mathscr{I}_{(t)_{0}} \otimes E\right)=h^{0}\left(E^{\vee} \otimes E\right)$. Thus the assertion immediately follows from (1.2).

(2) By the Kodaira vanishing theorem, we have $h^{i}\left(E^{\vee}\right)=0(i=0,1)$. So (2a) follows from (1). For (2b), we have

$$
\begin{aligned}
2 c_{2}(E)-s(E)= & \left(\operatorname{deg}\left(\left.M_{2}\right|_{D_{1}}\right)-h^{0}\left(M_{2}\right)+h^{0}\left(M_{1}^{-1} \otimes M_{2}\right)+1\right) \\
& +\left(\operatorname{deg}\left(M_{1} \mid D_{2}\right)-h^{0}\left(M_{1}\right)+h^{0}\left(M_{2}^{-1} \otimes M_{1}\right)+1\right) \\
\geq & \left(\operatorname{deg}\left(\left.M_{2}\right|_{D_{1}}\right)-h^{0}\left(\left.M_{2}\right|_{D_{1}}\right)+1\right)+\left(\operatorname{deg}\left(\left.M_{1}\right|_{D_{2}}\right)-h^{0}\left(\left.M_{1}\right|_{D_{2}}\right)+1\right) \\
\geq & \Delta\left(D_{1},\left.M_{2}\right|_{D_{1}}\right)+\Delta\left(D_{2},\left.M_{1}\right|_{D_{2}}\right) \geq 0 . \quad \square
\end{aligned}
$$

Lemma 2.3. Let $E$ be a vector bundle of rank 2 on a projective variety of dimension $n \geq 1$. Assume that $E$ has the following nontrivial extension for line bundles $L$ and $M$ :

$$
0 \longrightarrow M \stackrel{f}{\longrightarrow} E \stackrel{g}{\longrightarrow} L \longrightarrow 0
$$

(1) Assume that $h^{0}\left(M \otimes L^{-1}\right)=h^{0}\left(L \otimes M^{-1}\right)=0$. Then $h^{0}\left(E \otimes E^{\vee}\right)=1$.

(2) Assume that $h^{1}\left(M \otimes L^{-1}\right)=1$. Suppose that $h^{0}\left(E \otimes M^{-1}\right)=1$ (which holds whenever $\left.h^{0}\left(L \otimes M^{-1}\right)=0\right)$. Then $h^{0}\left(E \otimes E^{\vee}\right)=1+h^{0}\left(M \otimes L^{-1}\right)$. 
Proof. Since $(*)$ is nonsplit, the connecting homomorphism $\delta\left((*) \otimes L^{-1}\right)$ : $H^{0}(\mathscr{O}) \rightarrow H^{1}\left(M \otimes L^{-1}\right)$ for $(*) \otimes L^{-1}$ is injective. Thus $h^{0}\left(E \otimes L^{-1}\right)=$ $h^{0}\left(M \otimes L^{-1}\right)$, and therefore

$$
1 \leq h^{0}\left(E \otimes E^{\vee}\right) \leq h^{0}\left(E \otimes M^{-1}\right)+h^{0}\left(M \otimes L^{-1}\right)=1+h^{0}\left(M \otimes L^{-1}\right) .
$$

For (1), since $h^{0}\left(M \otimes L^{-1}\right)=0$, we have $h^{0}\left(E \otimes E^{\vee}\right)=1$.

To prove (2), it suffices to show that the connecting homomorphism $\delta\left(E \otimes(*)^{\vee}\right)$ is a zero map. We consider the diagram

$$
\begin{array}{ccc}
H^{0}(\mathscr{O}) & \stackrel{\delta\left(M \otimes(*)^{\vee}\right)}{\longrightarrow} H^{1}\left(M \otimes L^{-1}\right) \\
H^{0}\left(f \otimes M^{-1}\right) \downarrow & \\
H^{0}\left(E \otimes M^{-1}\right) \stackrel{\delta\left(E \otimes(*)^{\vee}\right)}{\longrightarrow} & H^{1}\left(E \otimes L^{-1}\right) .
\end{array}
$$

Since $\delta\left((*) \otimes L^{-1}\right): H^{0}(\mathscr{O}) \rightarrow H^{1}\left(M \otimes L^{-1}\right)$ is bijective by the assumption of (2), $H^{1}\left(f \otimes L^{-1}\right): H^{1}\left(M \otimes L^{-1}\right) \rightarrow H^{1}\left(E \otimes L^{-1}\right)$ is a zero map. Since $H^{0}\left(f \otimes M^{-1}\right)$ is isomorphic by assumption, $\delta\left(E \otimes(*)^{\vee}\right)$ is a zero map, as required.

The following proposition, which is a special case of a theorem of Fujita [11], plays an essential role for our classification in the case that $K \otimes \operatorname{det}(E)$ is not ample.

Proposition $2.4[11 ; 10,(20.8)]$. Let $E$ be a rank-2 ample vector bundle on a smooth projective surface $X$. Let $L=\operatorname{det}(E)$ and let $K$ be the canonical bundle of $X$. Then $K \otimes L$ is ample except the following cases:

(1) $(X, E) \cong\left(\mathbf{P}^{2}, \oplus^{2} \mathscr{O}(1)\right)$.

(2) $(X, E) \cong\left(\mathbf{P}^{2}, \mathscr{O}(1) \oplus \mathscr{O}(2)\right)$.

(3) $(X, E) \cong\left(\mathbf{P}^{2}, T_{\mathbf{P}^{2}}\right)$.

(4) There exists a vector bundle $\mathscr{F}$ on a smooth projective curve $C$ such that $X \cong \mathbf{P}_{C}(\mathscr{F})$ and $\left.E\right|_{F} \cong \bigoplus^{2} \mathscr{O}(1)$ for any fibre $F\left(\cong \mathbf{P}^{1}\right)$ of the projection $\pi: X \rightarrow C$.

The following lemma is used in our classification, to describe the structure of ample and spanned vector bundles on a geometrically ruled surface.

Lemma 2.5 [22, Lemma 2]. Let $\mathscr{E}$ be an ample and spanned vector bundle of rank $r \geq 2$ on a projective curve $C$. Take arbitrary points $p_{1}, \ldots, p_{r-1}$ of $C$ with $\mu_{i}=$ mult $_{p_{i}}(C)$.

(1) If $C$ is rational, then $c_{1}(\mathscr{E}) \geq\left(\sum_{i=1}^{r-1} \mu_{i}\right)+1$.

(2) If $C$ is nonrational, then $c_{1}(\mathscr{E}) \geq\left(\sum_{i=1}^{r-1} \mu_{i}\right)+2$.

\section{THE CASE OF $S(E)=2 c_{2}(E)$}

Our goal in this section is to prove the following theorem (see the classification chart (Figure 1)).

Theorem 3.1. Let $E$ be an ample and spanned rank-2 vector bundle on a smooth projective surface $X$. Then $s(E)=2 c_{2}(E)$ if and only if $(X, E)$ is isomorphic to one of the following:

(1) $\left(\mathbf{P}^{2}, \mathscr{O}(2) \oplus \mathscr{O}(2)\right)$. 
(2) $\left(\mathbf{P}^{2}, T_{\mathbf{P}}(1)\right)$, where $T_{\mathbf{P}}$ is the tangent bundle of $\mathbf{P}^{2}$.

(3) $\left(\mathbf{F}_{0}, \mathscr{O}(2 h+f) \oplus \mathscr{O}(h+2 f)\right)$.

(4) $\left(\mathbf{F}_{1}, E\right)$, where $E$ is a unique bundle defined by a nonsplit exact sequence

$$
0 \longrightarrow \mathscr{O}(2 h+2 f) \longrightarrow E \longrightarrow \mathscr{O}(h+3 f) \longrightarrow 0 .
$$

(5) $\left(\mathbf{P}^{2}, \mathscr{O}(1) \oplus \mathscr{O}(1)\right)$.

(6) $\left(\mathbf{P}^{2}, \mathscr{O}(1) \oplus \mathscr{O}(2)\right)$.

(7) $\left(\mathbf{P}^{2}, T_{\mathbf{P}}\right)$.

(8) $\left(\mathbf{F}_{e}, \mathscr{O}(h+a f)^{\oplus 2}\right)(a>e \geq 0)$.

(9) $\left(\mathbf{F}_{e}, \mathscr{O}(h+a f) \oplus \mathscr{O}(h+(a+1) f)\right)(a>e \geq 0)$.

Here, $\mathbf{F}_{e}$ denotes the eth Hirzebruch surface with the minimal section $h$ and a fibre $f$, and with the projection $\pi: \mathbf{F}_{e} \rightarrow \mathbf{P}^{1}$.

First we recall the following two fundamental results. For a proof, see [3].

Proposition 3.2 [3, (1.1)]. Let $E$ be an ample and spanned vector bundle of rank $n$ on a smooth projective variety of dimension $n$. Assume that $s(E)=n c_{n}(E)$. Then $X$ is rational.

Lemma 3.3 [3, (1.4)]. We keep the notation and the assumptions as in the theorem. Set $L=\operatorname{det}(E)$. Assume that $s(E)=2 c_{2}(E)$. Then:

(1) $g(X, L)=h^{0}(L \otimes K)<c_{2}(E)$;

(2) $h^{0}(L)=h^{0}(E)+c_{2}(E)-1 \geq 3 c_{2}(E)$.

Now we prove the theorem. Let $L$ be the determinant bundle of $E$. The proof consists of two part: One is the case $L \otimes K$ is ample, and the other is the case $L \otimes K$ is not ample.

First we treat the case $L \otimes K$ is ample.

Step 3.4. Assume that $s(E)=2 c_{2}(E)$ and that $L \otimes K$ is ample. Then $K^{2} \geq 8$, hence $X$ is isomorphic to $\mathbf{P}^{2}$ or $\mathbf{F}_{e}$.

Proof. First note that $K \otimes L$ is spanned. Indeed, if not, by [22, Theorem A] we would have $(X, E) \cong\left(\mathbf{P}^{2}, \mathscr{O}(1)^{\oplus 2}\right)$, hence $K \otimes L \cong \mathscr{O}(-1)$, contradiction. In particular $h^{0}(K \otimes L) \geq 3$. By (3.3)(1) and (2.1)(2), $c_{2}(E)>$ $g(\mathbf{P}(E), \mathscr{O}(1))=g(X, L) \geq 3$. Then, if $0 \leq \Delta:=\Delta(\mathbf{P}(E), \mathscr{O}(1)) \leq 2$, we would have $d(\mathbf{P}(E), \mathscr{O}(1)) \geq \Delta+2 c_{2}(E)-2 \geq 2 \Delta+1$ by (2.1)(3). So it follows from $[10,(3.5)]$ that $g(\mathbf{P}(E), \mathscr{O}(1))=\Delta \leq 2$, contradiction. Therefore we have $\Delta \geq 3$. Now we prove this step, according to $g(X, K \otimes L)$. If $g(X, K \otimes L)=0$, by [10, (12.1), (5.10)], then $X \cong \mathbf{P}^{2}$ or $\mathbf{F}_{e}$, hence $K^{2} \geq 8$, as required. If $g(X, K \otimes L)=1$, by [10, (12.3)], then $X$ is a Del Pezzo variety with $L=K^{\otimes-2}$. (For the definition of a Del Pezzo variety, see $[10,(6.3),(6.4)])$. By the Kodaira vanishing theorem and Riemann-Roch theorem, we have $h^{0}(L)=(1 / 2)\left(L^{2}-L . K\right)+1=3 K^{2}+1$ and $h^{0}(L \otimes K)=$ $(1 / 2)\left(L^{2}+L . K\right)+1=K^{2}+1$. So $3 K^{2}+1 \geq 3 c_{2}(E) \geq 3 K^{2}+6$ by (3.3), contradiction. If $g(X, K \otimes L) \geq 2$, then, by (2.1)(4), (3.3)(1), and by (3.2), we have $0 \leq(g(X, K \otimes L)-2)+(\Delta-3) \leq K^{2}-8$, hence $K^{2} \geq 8$. So, by (3.2), $\mathrm{X}$ is isomorphic to $\mathbf{P}^{2}$ or $\mathbf{F}_{e}$, as required.

Step 3.5. Assume that $X=\mathbf{P}^{2}$ and that $L \otimes K$ is ample. Then $s(E)=2 c_{2}(E)$ if and only if $(X, E)$ is isomorphic to (1) or (2). 
Proof. First we assume that $s(E)=2 c_{2}(E)$. Set $c_{1}=c_{1}(E)=\operatorname{deg}(L)$ and $c_{2}=c_{2}(E)$. Since $L$ and $L \otimes K$ are ample, we have $c_{1} \geq 4$ and it follows from (3.3) that

(i) $(1 / 2)\left(c_{1}+1\right)\left(c_{1}+2\right)=h^{0}(L) \geq 3 c_{2} \geq 3 h^{0}(L \otimes K)+3=(3 / 2)\left(c_{1}-1\right)\left(c_{1}-2\right)+3$.

Comparing the both sides, we have $0 \geq\left(c_{1}-1\right)\left(c_{1}-5\right)$, hence $c_{1}=4$ or 5 . If $c_{1}=4$, by (i), then $c_{2}=4$, or 5 . Also, if $c_{1}=5$, then $c_{2}=7$. Now we look at the possibilities of $E$ according to $c_{1}$ and $c_{2}$. In each case, if $t$ is a general global section with a 0 -dimensional smooth zero $Z:=(t)_{0}$, then we have the Koszul sequence

$$
0 \longrightarrow \mathscr{O} \longrightarrow E \longrightarrow \mathscr{I}_{Z} \otimes \mathscr{O}\left(c_{1}\right) \longrightarrow 0,
$$

and, since $s(E)=2 c_{2}(E)$, we may assume that, for each $0 \leq l \leq c_{1}$, $h^{0}\left(\mathscr{J}_{Z} \otimes \mathscr{O}(l)\right)=h^{0}(\mathscr{O}(l))-c_{2}$ if $h^{0}(\mathscr{O}(l)) \geq c_{2}, h^{0}\left(\mathscr{I}_{Z} \otimes \mathscr{O}(l)\right)=0$ otherwise.

Case $\left(c_{1}, c_{2}\right)=(4,4)$. By (ii), $h^{0}(E(-2))=h^{0}\left(\mathscr{J}_{Z} \otimes \mathscr{O}(2)\right)=2$ and $h^{0}(E(-3))=h^{0}\left(\mathscr{I}_{Z} \otimes \mathscr{O}(1)\right)=0$. Let $u$ be a nonzero global section of $E(-2)$. Then $\operatorname{dim}(u)_{0}=0, c_{1}(E(-2))=0$, and $c_{2}(E(-2))=0$. Hence there exists an exact sequence: $0 \rightarrow \mathscr{O} \rightarrow E(-2) \rightarrow \mathscr{O} \rightarrow 0$, which is split since $\operatorname{Ext}^{1}(\mathscr{O}, \mathscr{O})=0$. Hence $E \cong \mathscr{O}(2)^{\oplus 2}$, which is the bundle in (1).

Case $\left(c_{1}, c_{2}\right)=(4,5)$. By (3.3) $(2), h^{0}(E)=11$. Hence, by $(1.2), s(E)=$ $2 c_{2}(E)$ implies that $h^{0}\left(\mathscr{I}_{Z} \otimes E\right)=1$. By (ii), we have $h^{0}(E(-4))=0$. Tensoring (ii) by $E^{\vee} \cong E(-4)$, we have an exact sequence $0 \rightarrow E(-4) \rightarrow E \otimes E^{\vee} \rightarrow$ $\mathscr{I}_{Z} \otimes E \rightarrow 0$. So $h^{0}\left(E \otimes E^{\vee}\right)=1$, hence $E$ is simple. Therefore $E$ is stable since $E$ is of rank 2 (see [28, p. 172, (1.2.10)]). But $c_{1}(E(-2))=0$ and $h^{0}(E(-2))=h^{0}\left(\mathscr{I}_{Z} \otimes \mathscr{O}(2)\right)=1$ by (ii). This contradicts the stability of $E$.

Case $\left(c_{1}, c_{2}\right)=(5,7)$. By (ii), $h^{0}(E(-2))=h^{0}\left(\mathscr{I}_{Z} \otimes \mathscr{O}(3)\right)=3$ and $h^{0}(E(-3))=h^{0}\left(\mathscr{I}_{Z} \otimes \mathscr{O}(2)\right)=0$. A nonzero section $u$ of $E(-2)$ induces an exact sequence $0 \rightarrow \mathscr{O} \rightarrow E(-2) \rightarrow \mathscr{I}_{Q} \otimes \mathscr{O}(1) \rightarrow 0$ for some $Q \in \mathbf{P}^{2}$, where $\mathscr{I}_{Q}$ is the ideal sheaf of $\{Q\} \subset \mathbf{P}^{2}$. Then $h^{1}(E(-3))=h^{2}(E(-4))=0$, hence $E(-2)$ is 0-regular (see [26, Lecture 14, p. 99]). Therefore, $E(-2)$ is spanned. Since $h^{0}(E(-2))=3$, we have an exact sequence $0 \rightarrow \mathscr{O} \rightarrow$ $H^{0}(E(-2)) \otimes \mathscr{O}(1) \rightarrow E(-1) \rightarrow 0$. Thus $E(-1)$ is ample and spanned, hence uniform, so $E(-1) \cong T_{\mathbf{P}}$. This is the bundle in (2).

Conversely, we see that the bundles in (1) and (2) are ample and spanned with $K \otimes L$ ample and with $s(E)=2 c_{2}(E)$; the ampleness and spannedness are clear. For $s(E)$, using $(2.2)(1)$, one checks immediately that $s\left(\mathscr{O}(2)^{\oplus 2}\right)=$ $2 c_{2}\left(\mathscr{O}(2)^{\oplus 2}\right)$. For $(2)$, we have already seen that $s\left(T_{\mathbf{P}}(1)\right)=2 c_{2}\left(T_{\mathbf{P}}(1)\right)$ in $(1.5)(2)$.

Step 3.6. Assume that $X$ is an eth Hirzebruch surface $\mathbf{F}_{e}$, with the minimal section $h$ and a fibre $f$ and the projection $\pi: \mathbf{F}_{e} \rightarrow \mathbf{P}^{1}$. Assume that $L \otimes K$ is ample. Then $s(E)=2 c_{2}(E)$ if and only if $(X, E)$ is isomorphic to (3) or (4).

Proof. First we assume that $s(E)=2 c_{2}(E)$. Set $L=\mathscr{O}(a h+b f)$. Then $L \otimes K=\mathscr{O}((a-2) h+(b-e-2) f)$. First we claim that $\left(e, a, b, c_{2}\right)=$ $(0,3,3,5)$ or $(1,3,5,6)$. In fact, since $\left.\operatorname{deg} L\right|_{h} \geq 2$ by $(2.5)(1)$, we have

$$
b \geq a e+2 \text {. }
$$


Since $L$ and $L \otimes K$ are ample, it follows from (3.3) that

$$
\begin{aligned}
(a+1)(b-a e / 2+1) & =h^{0}(L) \geq 3 c_{2} \geq 3 h^{0}(L \otimes K)+3 \\
& =3(a-1)(b-a e / 2-1)+3 .
\end{aligned}
$$

Comparing the both sides, we have $3 \geq(a-2)(2 b-a e-4)$. Since $2 b-a e-4 \geq$ $a e$ by (i) and since $a \geq 3$ by the ampleness of $L \otimes K$, it follows that

$$
3 \geq(a-2)(2 b-a e-4) \geq(a-2) a e,
$$

hence $3 / a(a-2) \geq e$. Thus $e=0$, or 1 . If $e=0$, by (iii), then $3 \geq$ $2(a-2)(b-2)$, hence $a=3$ and $b=3$. Thus $c_{2}=5$, by (ii). This is the first case of the claim. If $e=1$, then $3 \geq(a-2) a$ by (iii), hence $a=3$. Then $3 \geq 2 b-7$ by (iii), and $b \geq 5$ by (i). Thus $b=5$, hence $c_{2}=6$ by (ii). This is the second case of the claim. In the both cases, since $a=3$ and since $E$ is ample and spanned, $\left.E\right|_{f} \cong \mathscr{O}_{\mathbf{P}^{1}}(1) \oplus \mathscr{O}_{\mathbf{P}^{1}}(2)$. By Grauert's theorem (see, for example, [18, III, 12.9]), $\pi_{*} E(-2)$ is a locally free sheaf of rank 1 and a natural map $\pi^{*} \pi_{*} E(-2) \rightarrow E(-2)$ makes $\pi^{*} \pi_{*} E(-2)$ a subbundle of $E(-2)$. Thus we have the following exact sequence for some integers $\alpha$ and $\beta$ :

$$
0 \longrightarrow \mathscr{O}(2 h+\alpha f) \longrightarrow E \longrightarrow \mathscr{O}(h+\beta f) \longrightarrow 0 \text {. }
$$

Then $c_{2}=-2 e+\alpha+2 \beta$ and $b=\alpha+\beta$, hence $\alpha=2 b-c_{2}-2 e$ and $\beta=c_{2}-b+2 e$.

Case $\left(e, a, b, c_{2}\right)=(0,3,3,5)$. Then $\alpha=1$ and $\beta=2$. Since $\operatorname{Ext}^{1}(\mathscr{O}(h+2 f), \mathscr{O}(2 h+f))=H^{1}(\mathscr{O}(h-f))=0$, we have $E=\mathscr{O}(h+2 f) \oplus$ $\mathscr{O}(2 h+f)$. This is the bundle in (3).

Case $\left(e, a, b, c_{2}\right)=(1,3,5,6)$. Then $\alpha=2$ and $\beta=3$. If the sequence (iv) were split, by $(2.2)(2)$, we would have $s(E)=11 \neq 2 c_{2}$. Hence (iv) is nonsplit. This is the bundle in (4).

Conversely, the bundles in (3) is clearly ample and spanned with $K \otimes L$ ample, and satisfies $s(E)=2 c_{2}(E)$ by (2.2)(2). The global exactness implies that the bundle (4) is spanned. Due to [14, (2.1)], the ampleness of (4) is checked by restricting to the integral curves (see also [3, 4, 25]). By (2.2) and $(2.3)(2), s(E)=2 c_{2}(E)$ holds for (4).

The remaining part of the classification is the case $L \otimes K$ is not ample.

Step 3.7. Assume that $L \otimes K$ is not ample. Then $s(E)=2 c_{2}(E)$ if and only if $(X, E)$ is isomorphic to (5), (6), (7), (8), or (9).

Proof. First we assume that $s(E)=2 c_{2}(E)$. Since $X$ is rational by (3.2), it follows from (2.4) that $(X, E)$ is one of $(5),(6),(7)$, or $\left(\mathbf{F}_{e}, E\right)$ with $\left.E\right|_{\pi^{-1}(x)} \cong$ $\mathscr{O}_{\mathbf{P}^{1}}(1)^{\oplus 2}$ for each fibre $\pi^{-1}(x) \cong \mathbf{P}^{1}$. If $(X, E)$ is the pair in the last case, by Grauert's theorem (see, for example, [18, III, 12.9]), $\pi_{*}(E(-1))$ is locally free of rank 2 and a natural homomorphism $\pi_{*} \pi^{*}(E(-1)) \rightarrow E(-1)$ is isomorphic. Since every vector bundle on $\mathbf{P}^{1}$ is a direct sum of line bundles (see, for example, [18, V, Example 2.6; 28, I, (2.1.1)]), $E \cong \mathscr{O}(h+a f) \oplus \mathscr{O}(h+b f)$ for some $a, b \in \mathbf{Z}$. Then $E$ is ample and spanned if and only if $a, b>e \geq 0[18, \mathrm{p}$. $382,(2.20)$ ]. By $(2.2), s(E)=4 a-2 e=2 c_{2}(E)$ if $a=b$, which are the bundles in (8). If $b>a$, then $s(E)=3 a+b-2 e+1$. Since $s(E)=2 c_{2}(E)=2(a+b-e)$, we have $b=a+1$, which are the bundles in (9). Conversely, we prove the "if" part. For (8) and (9), we are done. For the bundles in (5), (6), and (7), the 
ampleness and spannedness are clear. Thus we check that $s(E)=2 c_{2}(E)$; for $(5)$ and (6), by (2.2)(2), we have $s(E)=2$ for (5) and $s(E)=4$ for (6). For $(7), s(E)=6=2 c_{2}(E)$ as we have already seen in (1.5)(2).

\section{The CASE OF $s(E)=2 c_{2}(E)-1 ; K \otimes \operatorname{det}(E)$ AMPLE}

The following two sections are devoted to the proof of the following theorem (see the classification chart (Figure 2)).

Theorem 4.1. Let $E$ be an ample and spanned rank-2 vector bundle on a smooth projective surface. Then $s(E)=2 c_{2}(E)-1$ if and only if $(X, E)$ is isomorphic to one of the following:

(1) $\left(\mathbf{P}^{2}, \mathscr{O}(1) \oplus \mathscr{O}(3)\right)$.

(2) $\left(\mathbf{P}^{2}, F^{P}(2)\right)$, where $F^{P}$ is the vector bundle defined by an extension of the ideal sheaf $\mathscr{I}_{P}$ of $P \in \mathbf{P}^{2}$ by the structure sheaf $\mathscr{O}$ ([15, (1.33)]; see also [4, p. 484]), namely $0 \rightarrow \mathscr{O} \rightarrow F^{P} \rightarrow \mathscr{I}_{P} \rightarrow 0$ is exact.

(3) $\left(\mathbf{P}^{2}, \mathscr{O}(2) \oplus \mathscr{O}(3)\right)$.

(4) $\left(\mathbf{F}_{0}, \mathscr{O}(2 h+2 f) \oplus \mathscr{O}(h+f)\right)$.

(5) $\left(\mathbf{F}_{0}, \mathscr{O}(2 h+2 f) \oplus \mathscr{O}(h+2 f)\right)$.

(6) $\left(\mathbf{F}_{0}, \mathscr{O}(2 h+2 f) \oplus \mathscr{O}(h+3 f)\right)$.

(7) $\left(\mathbf{F}_{0}, E\right)$, where $E$ is a bundle defined by a nonsplit exact sequence

$$
0 \longrightarrow \mathscr{O}(2 h+f) \longrightarrow E \longrightarrow \mathscr{O}(h+3 f) \longrightarrow 0 .
$$

(8) $\left(\mathbf{F}_{1}, \mathscr{O}(2 h+3 f) \oplus \mathscr{O}(h+2 f)\right)$.

(9) $\left(\mathbf{F}_{1}, \mathscr{O}(2 h+3 f) \oplus \mathscr{O}(h+3 f)\right)$.

(10) $\left(\mathbf{F}_{1}, E\right)$, where $E$ is a unique bundle defined by a nonsplit exact sequence

$$
0 \longrightarrow \mathscr{O}(2 h+3 f) \longrightarrow E \longrightarrow \mathscr{O}(h+4 f) \longrightarrow 0 .
$$

(11) $\left(\mathbf{F}_{2}, E\right)$, where $E$ is a unique bundle defined by a nonsplit exact sequence

$$
0 \longrightarrow \mathscr{O}(2 h+4 f) \longrightarrow E \longrightarrow \mathscr{O}(h+4 f) \longrightarrow 0 .
$$

(12) $\left(\mathbf{F}_{e}, \mathscr{O}(h+a f) \oplus \mathscr{O}(h+(a+2) f)\right)(a>e \geq 0)$.

(13) $\left(\mathbf{P}_{C}(\mathscr{F}), \pi^{*}(\mathscr{E} \otimes \mathscr{A}) \otimes \mathscr{O}(1)\right)$, where $\mathbf{P}_{C}(\mathscr{F})$ is a geometrically ruled surface over an elliptic curve $C$ with the projection $\pi: \mathbf{P}_{C}(\mathscr{F}) \rightarrow C$ and with the tautological line bundle $\mathscr{O}(1), \mathscr{F}$ and $\mathscr{E}$ are normalized (i.e., $H^{0}(\mathscr{F}) \neq 0$ but $H^{0}(\mathscr{F} \otimes \mathscr{N})=0$ for every line bundle $\mathcal{N}$ on $C$ with $\operatorname{deg} \mathscr{N}<0)$ rank-2 vector bundles on $C$ of $\operatorname{deg} \mathscr{F}=-e$ and $\operatorname{deg} \mathscr{E}=1$, and $\mathscr{A}$ is a line bundle on $C$ of $\operatorname{deg} \mathscr{A}=\alpha$ with $\alpha-e \geq 1$.

First we recall Ballico's result. In order to prepare our notation, we give a proof here.

Proposition 4.2 [3, (1.2)]. Let $E$ be a spanned vector bundle of rank $n$ with $c_{n}(E)>0$ on a smooth projective variety $X$ of dimension $n \geq 2$. Assume that $s(E)=n c_{n}(E)-1$. Then $X$ is dominated by $\mathbf{P}_{C}(\mathscr{H})$, where $\mathscr{H}$ is a vector bundle of rank $h^{0}(X, E)-n\left(c_{n}(E)-1\right)$ on a (possibly noncomplete) integral curve $C$. In particular, $X$ is uniruled. 
Proof. Set $c=c_{n}(E)$ and $N+1=h^{0}(X, E)$. Let $X^{c-1}$ and $X^{c}$ be the $(c-1)$ time and $c$-time products of $X$, and $|E|=\mathbf{P}\left(H^{0}(E)^{\vee}\right)$. We consider incidence correspondences

$$
\begin{gathered}
F=\left\{\left([t],\left(P_{1}, \ldots, P_{c-1}\right)\right) ;[t] \in|E|, P_{i} \in X, t\left(P_{i}\right)=0\right\} \subseteq|E| \times X^{c-1}, \\
G=\left\{\left([u],\left(Q_{1}, \ldots, Q_{c}\right)\right) ;[u] \in|E|, Q_{i} \in X, u\left(Q_{i}\right)=0\right\} \subseteq|E| \times X^{c},
\end{gathered}
$$

and the following commutative diagram:

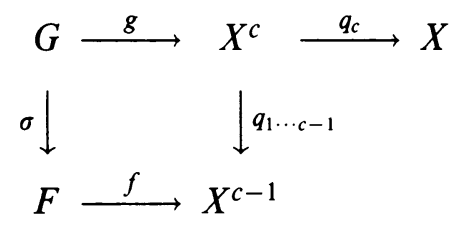

where $g, f$ are the second projections, $q_{c}$ the projection to $c$ th factor, and $q_{1 \cdots c-1}$ the projection to $(1, \ldots, c-1)$-factor. Note that $F$ is defined by scheme-theoretically by $F=\mathbf{P}_{X^{c-1}}(\mathscr{F})$ for a coherent sheaf

$$
\mathscr{F}=\operatorname{Coker}\left(\left(\bigoplus_{i=1}^{c-1} p_{i}^{*} e\right)^{\vee}: \bigoplus_{i=1}^{c-1} p_{i}^{*} E^{\vee} \rightarrow H^{0}(E)^{\vee} \otimes \mathscr{O}_{X^{c-1}}\right),
$$

where $e$ is the evaluation map $H^{0}(X, E) \otimes \mathscr{O}_{X} \rightarrow E, \vee$ denotes a dual, and $p_{i}$ the $i$ th projection $X^{c-1} \rightarrow X$, and that the same is true for $G$. Since $s(E)=n c-1$ and hence $H^{0}(E) \rightarrow \bigoplus_{i=1}^{c-1} E \otimes k\left(P_{i}\right)$ is surjective for some $\left(P_{1}, \ldots, P_{c-1}\right) \in X^{c-1}, \quad V_{1}:=X^{c-1} \backslash \operatorname{Supp}\left(\operatorname{Coker}\left(\bigoplus_{i=1}^{c-1} p_{i}^{*} e\right)\right)$ is nonempty open subset over which $f: F \rightarrow X^{c-1}$ is $\mathbf{P}^{N-c(n-1)}$-bundle. Let

$$
V_{2}=X^{c-1} \backslash \operatorname{diag} X^{c-1}=\left\{\left(P_{1}, \ldots, P_{c-1}\right) ; P_{i} \neq P_{j}(i \neq j)\right\} .
$$

For a nonempty open subset $W:=\left\{\left([t],\left(P_{1}, \ldots, P_{c-1}\right)\right) \in F ;(t)_{0}\right.$ is smooth, $t$ computes $s(E)$, and $\left.P_{i} \neq P_{j}(i \neq j)\right\}$ of $F$, since $\sigma^{-1}\left([t],\left(P_{1}, \ldots, P_{c-1}\right)\right) \cap$ $|E| \times\left(X^{c} \backslash \operatorname{diag} X^{c}\right)$ is one point $\left([t],\left(P_{1}, \ldots, P_{c}\right)\right)$ for $(t)_{0}=\left\{P_{1}, \ldots, P_{c}\right\}$, by Zariski main theorem, there is a section $\sigma^{-1}: W \hookrightarrow G$ of $\sigma$. Note that $W$ dominates $|E|$ and $X^{c-1}$. Since $f$ is flat over $V_{1}, V_{3}:=f(W) \subset V_{1} \cap V_{2}$ is a open subset. For $\left(P_{1}, \ldots, P_{c-1}\right) \in V_{3}$, let

$$
V\left(P_{1}, \ldots, P_{c-1}\right)=q_{c} g \sigma^{-1}\left(f^{-1}\left(\left(P_{1}, \ldots, P_{c-1}\right)\right) \cap W\right)^{-},
$$

namely the closure of set of points $Q \in X$ such that there exists $t \in H^{0}(E)$ computing $s(E)$ with smooth $(t)_{0}=\left\{P_{1}, \ldots, P_{c-1}, Q\right\}$. Then, since the dimension of general fibre $G \rightarrow g(G)$ is $N-s(E)$, a general fibre of $q_{1 \ldots c}$ is of dimension $n-1=(N-n(c-1))-(N-s(E))$. By Chevalley's theorem, $V_{4}:=\left\{\left(P_{1}, \ldots, P_{c-1}\right) \in V_{3} ; \operatorname{dim} V\left(P_{1}, \ldots, P_{c-1}\right)=n-1\right\}$ is a nonempty open of $X^{c-1}$. Set $V=V_{4}\left(=V_{1} \cap \cdots \cap V_{4}\right)$. Fix $\left(P_{1}, \ldots, P_{c-1}\right) \in V \subseteq X^{c-1}$. Then $V\left(P_{1}, \ldots, P_{c-1}\right) \neq V\left(Q_{1}, \ldots, Q_{c-1}\right)$ for some $\left(Q_{1}, \ldots, Q_{c-1}\right) \in V$. Indeed, $U=\left\{[t] \in|E| ;(t)_{0} \cap V\left(P_{1}, \ldots, P_{c-1}\right)=\varnothing\right\}$ is nonempty open, since $E$ is spanned of rank $n$ and hence the correspondence $T:=\{([t], P) ; t(P)=0\} \subseteq$ $|E| \times V\left(P_{1}, \ldots, P_{c-1}\right)$ is irreducible of $\operatorname{dim} T<\operatorname{dim}|E|$. Let $C$ be an integral curve on $V$ containing $\left(P_{1}, \ldots, P_{c-1}\right)$ and $\left(Q_{1}, \ldots, Q_{c-1}\right)$. Then $\mathscr{H}:=$ $\mathscr{F} \otimes \mathscr{O}_{C}$ is locally free of rank $h^{0}(X, E)-n\left(c_{n}(E)-1\right)$, and an induced rational $\operatorname{map} \mathbf{P}_{C}(\mathscr{H}) \hookrightarrow \mathbf{P}_{V_{1}}\left(\left.\mathscr{F}\right|_{V_{1}}\right) \rightarrow X$ is dominant, since $\operatorname{dim} V\left(Q_{1}, \ldots, Q_{c-1}\right)=$ $\operatorname{dim} V\left(P_{1}, \ldots, P_{c-1}\right)=n-1$ and $V\left(P_{1}, \ldots, P_{c-1}\right) \neq V\left(Q_{1}, \ldots, Q_{c-1}\right)$. 
Lemma 4.3. Let $E$ and $X$ as in (4.1). Set $L=\operatorname{det}(E)$. Assume that $s(E)=$ $2 c_{2}(E)-1$. Then $h^{0}(E) \leq h^{0}(L)-c_{2}(E)+1$ and $h^{0}(L) \geq 3 c_{2}(E)-1$.

Proof. The idea of this proof is found in Ballico [4, (1.3)]. We keep the notation as in (4.2). Set $\varphi=\phi_{|L|}: X \rightarrow \mathbf{P}\left(H^{0}(L)\right)$. First we note that there exists $\left(P_{1}, \ldots, P_{c-1}\right) \in V \subset X^{c-1}$ such that $P_{1}, \ldots, P_{c-1}$ are contained in a smooth and irreducible divisor $D \in|L|$ and such that $\varphi\left(P_{1}\right), \ldots, \varphi\left(P_{c-1}\right)$ are linearly independent in $\mathbf{P}\left(H^{0}(L)\right)$. Indeed, we consider an incidence correspondence $S:=\left\{\left(D,\left(P_{1}, \ldots, P_{c-1}\right)\right) ; D \in|L|, P_{1}, \ldots, P_{c-1} \in D\right\} \subseteq|L| \times X^{c-1}$ with the projection $h: S \rightarrow X^{c-1}$. Since $c_{2}(E)-1<2 c_{2}(E) \leq h^{0}(E) \leq h^{0}\left(\mathscr{I}_{(t)_{0}} \otimes L\right)+1 \leq$ $h^{0}(L)$ for any regular section $t$, there exists a nonempty open subset $V_{5} \subseteq V$ $\left(\subseteq X^{c-1}\right)$ over which $S$ is $\mathbf{P}^{m-c}$-bundle, where $m=h^{0}(L)$. Since every fibre of $S \rightarrow|L|$ is of dimension $c-1, h^{-1}\left(V_{5}\right)$ dominates $|L|$. Thus for the open subset $\mathscr{U}$ of $|L|$ of smooth, irreducible divisors, $S \cap\left(\mathscr{U} \times V_{5}\right)$ is nonempty as required. For such $\left(D,\left(P_{1}, \ldots, P_{c-1}\right)\right)$, we claim $\mathrm{Bs}\left|\mathscr{I}_{\left\{P_{1}, \ldots, P_{c-1}\right\}} \otimes L\right| \not \supset$ $V\left(P_{1}, \ldots, P_{c-1}\right)$ (where recall that $V\left(P_{1}, \ldots, P_{c-1}\right)$ is the closure of locus $Q \in$ $X$ for which there exists a regular global section $t$ vanishing at $P_{1}, \ldots, P_{c-1}, Q$ and computing $s(E))$; otherwise, $D$ and $V\left(P_{1}, \ldots, P_{c-1}\right)$ being irreducible, $D=V\left(P_{1}, \ldots, P_{c-1}\right)$ and hence $D$ would be a smooth rational curve, i.e., $g(X, L)=0$ : It follows from $(2.5)(1)$, together with [10, (12.1), (5.10)], that $(X, L) \cong\left(\mathbf{P}^{2}, \mathscr{O}(2)\right)$ and hence $E \cong \mathscr{O}(1)^{\oplus 2}$ : But this contradicts to $s(E)$ $=2 c_{2}(E)-1$. Thus, there exists a global section $t \in H^{0}(E)$ such that $(t)_{0} \backslash\left\{P_{1}, \ldots, P_{c-1}\right\} \notin \mathrm{Bs}\left|\mathcal{I}_{\left\{P_{1}, \ldots, P_{c-1}\right\}} \otimes L\right|, t$ vanishes at $P_{1}, \ldots, P_{c-1}$, and such that $t$ computes $s(E)$. Therefore

$$
h^{0}(L)-c_{2}(E) \leq h^{0}\left(\mathscr{J}_{(t)_{0}} \otimes L\right)<h^{0}\left(\mathscr{I}_{\left\{P_{1}, \ldots, P_{c-1}\right\}} \otimes L\right)=h^{0}(L)-c_{2}(E)+1,
$$

and consequently,

$$
2 c_{2}(E)=s(E)+1 \leq h^{0}(E) \leq h^{0}\left(\mathscr{J}_{\left(t_{0}\right.} \otimes L\right)+1=h^{0}(L)-c_{2}(E)+1
$$

by Koszul sequence, as required.

Remark 4.4. By the same argument as in the proof of (4.3), for any line bundle $M$ on $X$ with $h^{0}(M) \geq c_{2}(E)-1$ and for a given dense open subset $\mathscr{U}$ of $|M|$, there exists a dense open subset $V_{\mathscr{U}}$ of $X^{c_{2}-1}$ with the following property: For each $\left(P_{1}, \ldots, P_{c_{2}-1}\right) \in V_{\mathscr{U}}$, there exist a global section $t$ computing $s(E)$ with $(t)_{0}$ smooth and $D \in \mathscr{U}$ such that $h^{0}\left(\mathscr{J}_{(t)_{0}} \otimes M\right) \leq h^{0}\left(\mathscr{I}_{\left\{P_{1}, \ldots, P_{c_{2}-1}\right\}} \otimes M\right)=$ $h^{0}(M)-c_{2}(E)+1$ and $P_{1}, \ldots, P_{c_{2}-1} \in D$.

Now we prove the theorem. Set $L=\operatorname{det}(E)$.

Step 4.5. Assume that $s(E)=2 c_{2}(E)-1$. Then $K \otimes L$ is spanned.

Proof. We suppose to the contrary that $K \otimes L$ is not spanned. Then it follows from the result of [22] that $(X, E) \cong\left(\mathbf{P}^{2}, \mathscr{O}(1)^{\oplus 2}\right)$. This contradicts the assumption $s(E)=2 c_{2}(E)-1$.

In the remaining of this section, we treat the case that $K \otimes L$ is ample.

Step 4.6. Suppose that $s(E)=2 c_{2}(E)-1$ and that $K \otimes L$ is ample. Assume furthermore that $h^{0}(K \otimes L) \geq c_{2}(E)$. Then $g(X, K \otimes L)=0$ and $h^{0}(K \otimes L)=$ $c_{2}(E)$. Thus, by [10, (12.1), (5.10)], $X$ is isomorphic to $\mathbf{P}^{2}$ or $\mathbf{F}_{e}$.

Proof. The idea of this proof is found in Ballico [4, (1.2)]. Since $K \otimes L$ is ample and spanned by (4.5) and since $h^{0}(K \otimes L) \geq c_{2}(E)$, by (4.4), then 
there exist $\left(P_{1}, \ldots, P_{c_{2}-1}\right) \in V \subset X^{c_{2}-1}$ and a smooth irreducible divisor $D \in|K \otimes L|$ containing $P_{1}, \ldots, P_{c_{2}-1}$. Then the irreducible rational curve $V\left(P_{1}, \ldots, P_{c_{2}-1}\right)$ in the proof of (4.2) intersects with $D$ along a dense (open) subset of $V\left(P_{1}, \ldots, P_{c_{2}-1}\right)$, since the Cayley-Bacharach property [15, (1.33)] implies that $(t)_{0} \subset D$ for a regular global section $t$ with $(t)_{0} \supset\left\{P_{1}, \ldots, P_{c_{2}-1}\right\}$. Therefore $D=V\left(P_{1}, \ldots, P_{c_{2}-1}\right)$, so $D$ is a smooth rational curve, hence $g(X, K \otimes L)=0$. For the second, we assume to the contrary that $h^{0}(K \otimes L)>c_{2}(E)$. Then there exists a divisor $D^{\prime} \in|K \otimes L|$ with $D \neq D^{\prime}$ and passing through $P_{1}, \ldots, P_{c_{2}-1}$. Again the Cayley-Bacharach property implies that $D^{\prime} \supset V\left(P_{1}, \ldots, P_{c_{2}-1}\right)=D$, hence $D^{\prime}=D$. This is a contradiction to the choice of $D^{\prime}$.

Step 4.7. Suppose that $s(E)=2 c_{2}(E)-1$ and that $K \otimes L$ is ample. Assume furthermore that $h^{0}(K \otimes L) \leq c_{2}(E)-1$. Then $X$ is rational, and $K^{2} \geq 8$. Hence $X$ is isomorphic to $\mathbf{P}^{2}$ or $\mathbf{F}_{e}$.

Proof. First we claim that $X$ is rational. By (4.2) and $\operatorname{dim} X=2, X$ is ruled. If $X$ were nonrational ruled, we would have $q:=q(X) \geq 1$ and $K^{2} \leq 8-8 q$. Since $g(X, K \otimes L) \geq 0$ by [10, (12.3)], (2.1)(4) implies

$$
\begin{aligned}
0 & \leq \Delta\left(\mathbf{P}_{X}(E), \mathscr{O}(1)\right)+g(X, K \otimes L) \\
& \leq K^{2}+3\left(h^{0}(K \otimes L)-c_{2}(E)\right)+3 q+1 \leq 6-5 q .
\end{aligned}
$$

Hence $q=1$ and $\Delta\left(\mathbf{P}_{X}(E), \mathscr{O}(1)\right)=0$ or $g(X, K \otimes L)=0$ holds. When $\Delta\left(\mathbf{P}_{X}(E), \mathscr{O}(1)\right)=0, g\left(\mathbf{P}_{X}(E), \mathscr{O}(1)\right)=0$ by $[10,(12.1)]$, hence $g(X, L)=0$ by $(2.1)(3)$. Anyway, $X$ is a variety of sectional genus 0 , hence rational (see [10, (12.1), (5.10)]), contradiction. Thus $X$ is rational.

Since $K \otimes L$ is ample and spanned by the assumption and (4.5), we have $h^{0}(K \otimes L) \geq 3$. By (2.1)(1)(2) and our assumption, $c_{2}(E)>g\left(\mathbf{P}_{X}(E), \mathscr{O}(1)\right)=$ $g(X, L) \geq 3$. If $0 \leq \Delta:=\Delta\left(\mathbf{P}_{X}(E), \mathscr{O}(1)\right) \leq 2$, then

$$
d:=d\left(\mathbf{P}_{X}(E), \mathscr{O}(1)\right) \geq \Delta+2 c_{2}(E)-3 \geq 2 \Delta+1
$$

by (2.1)(3). So it follows from [10, (3.5)] that $g\left(\mathbf{P}_{X}(E), \mathscr{O}(1)\right)=\Delta \leq 2$, contradiction. Hence $\Delta \geq 3$. Note that, by the same argument, if $\Delta=3$ then $g(X, L)=g\left(\mathbf{P}_{X}(E), \mathscr{O}(1)\right)=3$. Now we show that $K^{2} \geq 8$ according to $g(X, K \otimes L)$. When $g(X, K \otimes L)=0, X$ is isomorphic to $\mathbf{P}^{2}$ or $\mathbf{F}_{e}$, and consequently, $K^{2} \geq 8$. When $g(X, K \otimes L)=1$, since $X$ is rational, $X$ is a Del Pezzo manifold with $K \otimes L=K^{-1}$. By (4.3) and our assumption, we have $3 K^{2}+2=h^{0}(L)+1 \geq 3 c_{2}(E) \geq 3 h^{0}(K \otimes L)+3=3 K^{2}+6$, contradiction (see (3.4)). When $g(X, K \otimes L) \geq 2,(2.1)(4)$ implies that

$$
0 \leq\left(\Delta\left(\mathbf{P}_{X}(E), \mathscr{O}(1)\right)-3\right)+(g(X, K \otimes L)-2) \leq K^{2}-7 .
$$

We claim that $K^{2} \geq 8$. To the contrary, we assume that $K^{2}=7$. By the inequality above, we have $g(X, K \otimes L)=2, \Delta\left(\mathbf{P}_{X}(E), \mathscr{O}(1)\right)=3$, hence $g(X, L)=3$ as remarked above, hence $(K \otimes L)^{2}=3$. So $K \otimes L$ is an ample line bundle on $X$ with $g(X, K \otimes L)=2$ and $(K \otimes L)^{2}=3$. However, the variety with the numerical data above does not appear in the classification table of surfaces of sectional genus $2[10,(15.2)]$. Therefore $K^{2} \geq 8$, hence $X$ is isomorphic to $\mathbf{P}^{2}$ or $\mathbf{F}_{e}$. 
Step 4.8. Assume that $X$ is a projective space $\mathbf{P}^{2}$ and that $K \otimes L$ is ample. Then $s(E)=2 c_{2}(E)-1$ if and only if $(X, E)$ is isomorphic to (1), (2), or (3). Proof. First we assume that $s(E)=2 c_{2}(E)-1$. Set $c_{1}=c_{1}(E)$ and $c_{2}=c_{2}(E)$. Since $L$ and $K \otimes L$ are ample, it follows from (4.3) and (4.6) that

(i) $(1 / 2)\left(c_{1}+1\right)\left(c_{1}+2\right)+1=h^{0}(L)+1 \geq 3 c_{2} \geq 3 h^{0}(K \otimes L)=(3 / 2)\left(c_{1}-1\right)\left(c_{1}-2\right)$.

Comparing both sides, we have $8 \geq\left(c_{1}-3\right)^{2}$. Since $c_{1} \geq 4, c_{1}=4$, or 5 . When $c_{1}=4, c_{2}=3,4$, or 5 by (i). When $c_{1}=5, c_{2}=6$ or 7 . In each case, if $t$ is a general section of $E$ with a 0 -dimensional smooth zero $Z:=(t)_{0}$, then we have Koszul sequence:

$$
0 \longrightarrow \mathscr{O} \longrightarrow E \longrightarrow \mathscr{I}_{Z} \otimes \mathscr{O}\left(c_{1}\right) \longrightarrow 0
$$

and we may assume that, for a point $P$ of $Z$, the points of $Z^{\prime}:=Z \backslash\{P\}$ enjoy the independence condition with respect to $\mathscr{O}(l)\left(0 \leq l \leq c_{2}\right)$ (see (4.4)). Now we will look at the possibilities of $E$, according to the value of $c_{1}$ and $c_{2}$.

Case $\left(c_{1}, c_{2}\right)=(4,3)$. By (ii), $h^{0}(E(-4))=h^{0}\left(\mathscr{J}_{Z}\right)=0$ and $h^{0}(E(-3))=$ $h^{0}\left(\mathscr{I}_{Z} \otimes \mathscr{O}(1)\right)=h^{0}\left(\mathscr{I}_{Z} \otimes K \otimes L\right)$. Since $\# Z=c_{2}=3$ and $h^{0}(K \otimes L)=3$, by the Cayley-Bacharach property of $Z$ with respect to $K \otimes L[15,(1.33)]$, $h^{0}\left(\mathscr{I}_{Z} \otimes K \otimes L\right) \geq 1$, hence $h^{0}(E(-3)) \geq 1$. A nonzero section $u \in H^{0}(E(-3))$ induces an exact sequence $0 \rightarrow \mathscr{O} \rightarrow E(-3) \rightarrow \mathscr{O}(-2) \rightarrow 0$, which is split, and hence, $E \cong \mathscr{O}(1) \oplus \mathscr{O}(3)$. This is the bundle in (1).

Case $\left(c_{1}, c_{2}\right)=(4,4)$. By (ii), $h^{0}(E(-2))=h^{0}\left(\mathscr{I}_{Z} \otimes \mathscr{O}(2)\right) \geq 2$ and $h^{0}(E(-3))=h^{0}\left(\mathscr{I}_{Z} \otimes \mathscr{O}(1)\right) \leq h^{0}\left(\mathscr{J}_{Z^{\prime}} \otimes \mathscr{O}(1)\right)=0$. By the same way as above (see also (3.5)), we have $E \cong \mathscr{O}(2)^{\oplus 2}$. But $s(E)=2 c_{2}(E)$, contradiction.

Case $\left(c_{1}, c_{2}\right)=(4,5)$. By (ii), $h^{0}(E(-2))=h^{0}\left(\mathscr{I}_{Z} \otimes \mathscr{O}(2)\right) \geq 1$ and $h^{0}(E(-3))=h^{0}\left(\mathscr{I}_{Z} \otimes \mathscr{O}(1)\right) \leq h^{0}\left(\mathscr{I}_{Z^{\prime}} \otimes \mathscr{O}(1)\right)=0$. A nonzero section $u \in$ $H^{0}(E(-3))$ induces an exact sequence $0 \rightarrow \mathscr{O} \rightarrow E(-2) \rightarrow \mathscr{I}_{P} \rightarrow 0$ for some $P \in \mathbf{P}^{2}$. Thus $E \cong F^{P} \otimes \mathscr{O}(2)$. This is the bundle in (2).

Case $\left(c_{1}, c_{2}\right)=(5,6)$. By (ii), $h^{0}(E(-3))=h^{0}\left(\mathscr{I}_{Z} \otimes \mathscr{O}(2)\right)=h^{0}\left(\mathscr{I}_{Z} \otimes K \otimes L\right)$ and $h^{0}(E(-4))=h^{0}\left(\mathscr{I}_{Z} \otimes \mathscr{O}(1)\right) \leq h^{0}\left(\mathscr{I}_{Z^{\prime}} \otimes \mathscr{O}(1)\right)=0$. By the CayleyBacharach property, $h^{0}\left(\mathscr{I}_{Z} \otimes K \otimes L\right) \geq 1$, hence $h^{0}(E(-3)) \geq 1$. Thus a nonzero section $u \in H^{0}(E(-3))$ induces an exact sequence $0 \rightarrow \mathscr{O} \rightarrow E(-3) \rightarrow$ $\mathscr{O}(-1) \rightarrow 0$, which is split since $\operatorname{Ext}^{1}(\mathscr{O}(-1), \mathscr{O})=0$. Thus $E \cong \mathscr{O}(2) \oplus \mathscr{O}(3)$. This is the bundle in (3).

Case $\left(c_{1}, c_{2}\right)=(5,7)$. By (ii), $h^{0}(E(-2))=h^{0}\left(\mathscr{I}_{Z} \otimes \mathscr{O}(3)\right) \geq 3$ and $h^{0}(E(-3))=h^{0}\left(\mathscr{I}_{Z} \otimes \mathscr{O}(2)\right) \leq h^{0}\left(\mathscr{I}_{Z^{\prime}} \otimes \mathscr{O}(2)\right)=0$. By the same way as in (3.5), we have $E \cong T_{\mathbf{P}}(1)$ and $s(E)=c_{2}(E)$, contradiction.

Conversely, by $(2.2)(2)$, one checks easily that the bundles in (1) and (3) satisfy the required property. In the case (2), by construction of $F^{P}, F^{P}$ has a surjection $\mathscr{O} \oplus \mathscr{O}(-1)^{\oplus 2} \rightarrow F^{P}$, and hence $F^{P}(2)$ is ample and spanned. We calculate $s(E)$ (cf. [4, (5.2)]). By the exact sequence defining $F^{P}$ and an exact sequence $0 \rightarrow F^{P} \rightarrow F^{P} \otimes F^{P} \rightarrow \mathscr{I}_{P} \otimes F^{P} \rightarrow 0$, we have $h^{0}\left(F^{P} \otimes F^{P}\right)=$ $h^{0}\left(\mathscr{J}_{P} \otimes F^{P}\right)+1$. From Koszul complex of $k(P)$ over $\mathscr{O}_{P}$, we have $0 \rightarrow \mathscr{I}_{P} \rightarrow$ $F^{P} \otimes \mathscr{I}_{P} \rightarrow \mathscr{I}_{P} \otimes \mathscr{I}_{P} \rightarrow 0$ and $0 \rightarrow k(P) \rightarrow \mathscr{I}_{P} \otimes \mathscr{I}_{P} \rightarrow \mathscr{I}_{P}^{2} \rightarrow 0$, and hence $h^{0}\left(F^{P} \otimes F^{P}\right)=2$. For a regular global section $t$ of $E$ with $P \notin Z:=(t)_{0}$, $0 \rightarrow \mathscr{I}_{Z}(2) \rightarrow E \otimes \mathscr{I}_{Z} \rightarrow \mathscr{I}_{P} \cdot \mathscr{I}_{Z}(2) \rightarrow 0$ is exact, and hence $h^{0}\left(E \otimes \mathscr{I}_{Z}\right) \leq$ $h^{0}\left(\mathscr{I}_{Z}(2)\right)+h^{0}\left(\mathscr{I}_{P} \cdot \mathscr{I}_{Z}(2)\right) \leq 2 h^{0}\left(\mathscr{I}_{Z}(2)\right)$. From Koszul sequence of $E$, we have $0 \rightarrow \mathscr{O}(-2) \rightarrow F^{P} \rightarrow \mathscr{I}_{Z}(2) \rightarrow 0$ and $0 \rightarrow F^{P}(-2) \rightarrow F^{P} \& F^{P} \rightarrow$ 
$\mathscr{I}_{Z} \otimes E \rightarrow 0$. By the first, $h^{0}\left(\mathscr{I}_{Z}(2)\right)=1$ hence $h^{0}\left(E \otimes \mathscr{J}_{Z}\right) \leq 2$. By the second, $h^{0}\left(\mathscr{I}_{Z} \otimes E\right) \geq h^{0}\left(F^{P} \otimes F^{P}\right)=2$. Thus $s(E)=h^{0}(E)-h^{0}\left(\mathscr{I}_{Z} \otimes E\right)=9$, as required.

Step 4.9. Assume that $X$ is an eth Hirzebruch surface $\mathbf{F}_{e}(e \geq 0)$ with the projection $\pi: \mathbf{F}_{e} \rightarrow \mathbf{P}^{1}$, the minimal section $h$, and a fibre $f$. Assume that $K \otimes L$ is ample. Then $s(E)=2 c_{2}(E)-1$ if and only if $(X, E)$ is isomorphic to (4), (5), (6), (7), (8), (9), (10), or (11).

Proof. First we assume that $s(E)=2 c_{2}(E)-1$. Set $L=\mathscr{O}(a h+b f)$, hence $K \otimes L=\mathscr{O}((a-2) h+(b-2-e) f)$. By (2.5)(1), we have $\left.\operatorname{deg} L\right|_{h} \geq 2$, hence

$$
b \geq a e+2 .
$$

Since $L$ and $K \otimes L$ are ample, it follows from (4.3) and (4.6) that

$$
\begin{aligned}
(a+1)(b-a e / 2+1)+1 & =h^{0}(L)+1 \geq 3 c_{2} \\
& \geq 3 h^{0}(K \otimes L)=3(a-1)(b-a e / 2-1) .
\end{aligned}
$$

Comparing both sides, we have $7 \geq(a-2)(2 b-a e-4)$. Since $2 b-a e \geq a e+4$ by (i), we have

$$
7 \geq(a-2)(2 b-a e-4) \geq a e(a-2) \geq 0 .
$$

Thus $7 / a(a-2) \geq e$. Since $a, b \geq 3$, we have $7 / 3 \geq 2 \geq e \geq 0$.

When $e=0,7 \geq(a-2)(2 b-4)$ by (iii), hence $(a, b)=(3,3),(3,4)$, $(3,5),(4,3),(5,3)$. Since $e=0$, replacing one projection with the other, we have only to consider the cases $(3,3),(3,4),(3,5)$.

When $e=1,7 \geq a(a-2)$ by (iii). Hence $a=3$ by the ampleness of $K \otimes L$. By (iii) and (i), $7 \geq b \geq 5$, and hence, we have $(a, b)=(3,5),(3,6),(3,7)$.

When $e=2,7 \geq 2 a(a-2)$ by (iii). Hence $a=3$ by the ampleness of $K \otimes L$. By (iii) and (i), $b=8$, and hence, we have $(a, b)=(3,8)$.

For each case, using (ii), we calculate $c_{2}$. Moreover, since $a=3$ and $E$ is ample and spanned, we have the following exact sequence for some integers $\alpha$ and $\beta$ (see (3.6)):

$$
0 \longrightarrow \mathscr{O}(2 h+\alpha f) \longrightarrow E \longrightarrow \mathscr{O}(h+\beta f) \longrightarrow 0 \text {. }
$$

Note that $c_{2}=-2 e+\alpha+2 \beta, b=\alpha+\beta$, hence $\beta=c_{2}-b+2 e, \alpha=2 b-c_{2}-2 e$, and $\alpha-\beta-e=3 b-2 c_{2}-5 e$. Since

$$
\begin{aligned}
\operatorname{Ext}^{1}(\mathscr{O}(h+\beta f), \mathscr{O}(2 h+\alpha f)) \cong H^{1}(\mathscr{O}(h+(\alpha-\beta) f)) \\
\cong H^{1}\left(\mathscr{O}_{\mathbf{P}^{1}}(\alpha-\beta) \oplus \mathscr{O}_{\mathbf{P}^{1}}(\alpha-\beta-e)\right)
\end{aligned}
$$

if $\alpha-\beta-e \geq-1$ then (iv) is split. If (iv) is split, $\mathscr{O}(2 h+\alpha f)$ and $\mathscr{O}(h+\beta f)$ are ample and spanned, hence, in the case $\left(e, a, b, c_{2}\right)=(1,3,5,6),(2,3,8,8)$, the exact sequences (iv) are nonsplit. Thus we have the following possibilities: 


$\begin{array}{ccccccccc}e & a & b & c_{2} & \alpha-\beta-e & \alpha & \beta & \text { (iv) is split? } & \text { type } \\ 0 & 3 & 3 & 4 & 1 & 2 & 1 & \text { split } & (4) \\ 0 & 3 & 3 & 5 & -1 & 1 & 2 & \text { split } & - \\ 0 & 3 & 4 & 6 & 0 & 2 & 2 & \text { split } & (5) \\ 0 & 3 & 4 & 7 & -2 & 1 & 3 & \text { split/nonsplit } & -/(7) \\ 0 & 3 & 5 & 8 & -1 & 2 & 3 & \text { split } & (6) \\ 1 & 3 & 5 & 5 & 0 & 3 & 2 & \text { split } & (8) \\ 1 & 3 & 5 & 6 & -2 & 2 & 3 & \text { nonsplit } & - \\ 1 & 3 & 6 & 7 & -1 & 3 & 3 & \text { split } & (9) \\ 1 & 3 & 7 & 9 & -2 & 3 & 4 & \text { split/nonsplit } & -/(10) \\ 2 & 3 & 8 & 8 & -2 & 4 & 4 & \text { nonsplit } & (11)\end{array}$

Now we claim that the split case in $\left(e, a, b, c_{2}\right)=(0,3,3,5),(0,3,4,7)$, and $(1,3,7,9)$ do not occur, and that $\left(e, a, b, c_{2}\right)=(1,3,5,6)$ case does not occur. Indeed, if the exact sequence (iv) is split in each case $\left(e, a, b, c_{2}\right)=$ $(0,3,3,5),(0,3,4,7),(1,3,7,9)$, using $(2.2)$, then it is easy to see that $s(E) \neq 2 c_{2}(E)-1$. If $\left(e, a, b, c_{2}\right)=(1,3,5,6)$, then (iv) is nonsplit. By $(3.1)(4), s(E)=12=2 c_{2}$. This is contradiction. Thus $E$ is one of (4) ,.., (11).

Conversely, it is easily checked that the bundles $E$ in $(4), \ldots,(11)$ satisfy the required properties, as in (3.6).

\section{The CASE OF $s(E)=2 c_{2}(E)-1 ; K \otimes \operatorname{det}(E)$ NOT AMPLE}

Step 5.1. Assume that $K \otimes L$ is not ample and that $s(E)=2 c_{2}(E)-1$. Then $X$ is a geometrically ruled surface $\mathbf{P}_{C}(\mathscr{F})$ over a smooth projective curve $C$ of genus $q=q(X)$ with the projection $\pi: X \rightarrow C$ and with the tautological line bundle $\mathscr{O}(1)$, and $E=\pi^{*}(\mathscr{E} \otimes \mathscr{A}) \otimes \mathscr{O}(1)$, where $\mathscr{F}$ and $\mathscr{E}$ are normalized rank-2 vector bundles on $C$ of degree $-e$ and $\beta$ respectively (i.e., $H^{0}(\mathscr{F}) \neq 0$ but $H^{0}(\mathscr{F} \otimes \mathscr{N})=0$ for every line bundle $\mathscr{N}$ on $C$ with $\left.\operatorname{deg} \mathscr{N}<0\right)$, and $\mathscr{A}$ is a line bundle on $C$ of $\operatorname{deg} \mathscr{A}=\alpha$. Since $\mathscr{E}$ and $\mathscr{F}$ are normalized, they fit into the following exact sequences (see [18, pp. 376-377, (2.12)(2.15)]):

$$
\begin{aligned}
& 0 \longrightarrow \mathscr{O}_{C} \longrightarrow \mathscr{E} \longrightarrow \mathscr{M} \longrightarrow 0, \\
& 0 \longrightarrow \mathscr{O}_{C} \longrightarrow \mathscr{F} \longrightarrow \mathscr{L} \longrightarrow 0,
\end{aligned}
$$

where $\mathscr{M}$ and $\mathscr{L}$ are line bundles on $C$. Consequently, there is an exact sequence:

$$
0 \longrightarrow \pi^{*}(\mathscr{A}) \otimes \mathscr{O}(1) \longrightarrow E \longrightarrow \pi^{*}(\mathscr{A} \otimes \mathscr{M}) \otimes \mathscr{O}(1) \longrightarrow 0 .
$$

Hence $c_{2}(E)=2 \alpha+\beta-e=\operatorname{deg}\left(\mathscr{L} \otimes \mathscr{M} \otimes \mathscr{A}^{\otimes 2}\right)$.

Proof. By (2.4), (X,E) is isomorphic to one of (1), (2), (3), (4) in (2.4). But in the cases (1), (2), and (3), we have $s(E)=2 c_{2}(E)$. Thus $(X, E)$ is the case (4). The rest follows from Grauert's theorem as in (3.7).

Step 5.2. We keep the notation and the assumptions as in (5.1). Assume furthermore that $c_{2}(E) \geq 3$. Then $h^{0}(K \otimes L)=q-1+c_{2}(E) \leq c_{2}(E)$, and consequently $q=q(X) \leq 1$. 
Proof. Set $\mathscr{N}=\mathscr{L} \otimes \mathscr{M} \otimes \mathscr{A}^{\otimes 2}$. For the canonical sheaf $\omega_{C}$ of $C$, since $\operatorname{deg}\left(\omega_{C} \otimes \mathscr{N}\right)=2 q-2+c_{2}(E) \geq 2 q+1$ and since $K \otimes L=\pi^{*}\left(\omega_{C} \otimes \mathscr{N}\right), \omega_{C} \otimes \mathscr{N}$ is very ample and $h^{0}(X, K \otimes L)=h^{0}\left(C, \omega_{C} \otimes \mathcal{N}\right)=q-1+c_{2}(E)$. Thus $\varphi:=$ $\phi_{|K \otimes L|}$ factors through the embedding $\psi:=\phi_{\left|\omega_{C} \otimes \mathcal{N}\right|}: C \rightarrow \mathbf{P}\left(H^{0}\left(C, \omega_{C} \otimes \mathscr{N}\right)\right)$, namely $\varphi=\psi \circ \pi$. To show $h^{0}(K \otimes L) \leq c_{2}(E)$, we suppose to the contrary that $h^{0}(K \otimes L) \geq c_{2}(E)+1$. As remarked in (4.4) (and (4.2)), there exist $\left(P_{1}, \ldots, P_{c_{2}-1}\right) \in V \subseteq X^{c_{2}-1}$, a (general) hyperplane $H$ of $\mathbf{P}\left(H^{0}(K \otimes L)\right)$, and a global section $t \in H^{0}(E)$ computing $s(E)$ such that $\varphi\left(P_{1}\right), \ldots, \varphi\left(P_{c_{2}-1}\right) \in$ $H,(t)_{0}=\left\{P_{1}, \ldots, P_{c_{2}-1}, P_{c_{2}}\right\}$ for some $P_{c_{2}} \in X$, and such that for $H$ the general position lemma of $\psi(C)=\varphi(X)$ holds, i.e., any distinct $c_{2}(E) \quad(\leq$ $\left.h^{0}\left(\omega_{c} \otimes \mathscr{N}\right)\right)$ points of $\psi(C) \cap H$ are linearly independent (see, for example, $\left[19\right.$, Proposition 5]). Note that $\pi\left(P_{i}\right) \neq \pi\left(P_{j}\right)$ if $i \neq j$, since $\left.E\right|_{F} \cong \mathscr{O}_{\mathbf{P}^{1}}(1)^{\oplus 2}$ for every fibre $F$ of $\pi$. Hence $\varphi\left(P_{i}\right) \neq \varphi\left(P_{j}\right)$ if $i \neq j$. So $\varphi\left(P_{1}\right), \ldots, \varphi\left(P_{c_{2}}\right)$ are linearly independent, since they lie on $H$ by the Cayley-Bacharach property $[15,(1.33)]$. But, by the Cayley-Bacharach property again, every hyperplane through $\varphi\left(P_{1}\right), \ldots, \varphi\left(P_{c_{2}-1}\right)$ is also through $\varphi\left(P_{c_{2}}\right)$, hence the linear subspace spanned by $\varphi\left(P_{1}\right), \ldots, \varphi\left(P_{c_{2}}\right)$ is of dimension $c_{2}-2$. This is contradiction. Thus we have $h^{0}(K \otimes L) \leq c_{2}(E)$ and hence $q \leq 1$.

Step 5.3. We keep the notation and the assumption as in (5.1). Suppose that $c_{2}(E)=2$. Then $q=q(X) \leq 1$.

Here, without any assumption on $s(E)$, we prove the following.

Proposition 5.4. Let $X$ be a geometrically ruled surface over a smooth projective curve $C$ with the projection $\pi: X \rightarrow C$ and let $E$ be an ample and spanned rank-2 vector bundle on $X$ with $c_{2}(E)=2$. Let $L=\operatorname{det}(E)$ and let $K$ be the canonical bundle of $X$. Suppose that $K \otimes L$ is not ample. Then the geometric genus $q$ of this $C$ is less than or equal to 1 .

To prove this, we need the following two lemmas.

Lemma 5.5. Let $\mathscr{G}$ be a normalized rank-2 vector bundle on a smooth projective curve $C$ and $\mathscr{B}$ a line bundle on $C$. Assume that $h^{0}(C, \mathscr{G} \otimes \mathscr{B}) \geq 3$. (This condition holds whenever $\mathscr{G} \otimes \mathscr{B}$ is ample and spanned.) Then $\operatorname{deg} \mathscr{B} \geq 1$.

Proof. Fix a point $x$ of $C$. Since rank $\mathscr{G} \otimes \mathscr{B}=2$ and $h^{0}(C, \mathscr{G} \otimes \mathscr{B}) \geq 3$, $h^{0}(C, \mathscr{G} \otimes \mathscr{B}(-x)) \geq 1$. Since $\mathscr{G}$ is normalized, det $\mathscr{B}(-x) \geq 0$, hence $\operatorname{deg} \mathscr{B} \geq 1$, as required.

Lemma 5.6. Let $\mathscr{L}$ be a special line bundle on a hyperelliptic curve $C$ of genus $g \geq 2$ (i.e., $\left.h^{i}(\mathscr{L}) \neq 0(i=0,1)\right)$. Then the complete linear system $g_{d}^{r}:=|\mathscr{L}|$ is of the form $r g_{2}^{1}+p_{1}+\cdots+p_{d-2 r}$, where no two of the $p_{i}$ 's are conjugate under the hyperelliptic involution. In particular, if $\mathscr{L}$ is a spanned and special line bundle on $C$, then $\operatorname{deg} \mathscr{L} \equiv 0(\bmod 2)$.

Proof. See, for example, [1, p. 41, Example D-9].

Proof of Proposition 5.4. Assume to the contrary that $q \geq 2$. Since $K \otimes L$ is not ample, by (2.4), we may assume that $X=\mathbf{P}_{C}(\mathscr{F})$ and $E=\pi^{*}(\mathscr{E} \otimes \mathscr{A}) \otimes \mathscr{O}(1)$, where $\mathscr{F}$ and $\mathscr{E}$ are normalized rank-2 vector bundles on $C$ of $\operatorname{deg} \mathscr{F}=\beta$ and $\operatorname{deg} \mathscr{E}=-e, \mathscr{A}$ is a line bundle of degree $\alpha$, and $\mathscr{O}(1)$ is the tautological 
line bundle of $\mathbf{P}_{C}(\mathscr{F})$. Since $\mathscr{F}$ and $\mathscr{E}$ are normalized, they fit into the exact sequences $0 \rightarrow \mathscr{O}_{C} \rightarrow \mathscr{E} \rightarrow \mathscr{M} \rightarrow 0$ and $0 \rightarrow \mathscr{O}_{C} \rightarrow \mathscr{F} \rightarrow \mathscr{L} \rightarrow 0$, where $\mathscr{M}$ and $\mathscr{L}$ are line bundles on $C$ of $\operatorname{deg} \mathscr{M}=\beta$ and $\operatorname{deg} \mathscr{L}=-e$. Thus $E$ fits into the following exact sequence:

$$
0 \longrightarrow \pi^{*} \mathscr{A} \otimes \mathscr{O}(1) \longrightarrow E \longrightarrow \pi^{*}(\mathscr{A} \otimes \mathscr{M}) \otimes \mathscr{O}(1) \longrightarrow 0 .
$$

First we claim that $C$ is a hyperelliptic curve. Set $\mathscr{N}=\mathscr{L} \otimes \mathscr{M} \otimes \mathscr{A} \otimes 2$. Since $K \otimes L=\pi^{*}\left(\omega_{C} \otimes \mathscr{N}\right)$ and since $\operatorname{deg}\left(\omega_{C} \otimes \mathscr{N}\right)=2 q-2+c_{2}(E)=2 q, \omega_{C} \otimes \mathscr{N}$ is spanned and so is $K \otimes L$, and hence a morphism $\phi_{|K \otimes L|}: X \rightarrow \mathbf{P}\left(H^{0}(K \otimes L)\right)$ factors through $\psi:=\phi_{\left|\omega_{C} \otimes \mathscr{N}\right|}: C \rightarrow \mathbf{P}\left(H^{0}(C, \omega \otimes \mathscr{N})\right)$. Namely $\phi_{|K \otimes L|}=$ $\psi \circ \pi$. Let $t \in H^{0}(E)$ be a regular global section and let $\left\{P_{1}, P_{2}\right\}=(t)_{0}$. Since $\left.E\right|_{F} \cong \mathscr{O}_{\mathbf{P I}^{1}}(1)^{\oplus 2}$ for any fibre $F$ of $\pi, \pi\left(P_{1}\right) \neq \pi\left(P_{2}\right)$. But by the Cayley-Bacharach property [15, (1.33)], $\psi \circ \pi\left(P_{1}\right)=\psi \circ \pi\left(P_{2}\right)$. Thus $\psi: C \rightarrow$ $\psi(C)$ is a finite morphism of degree $l \geq 2$. Since $h^{0}\left(\psi(C), \mathscr{O}_{\psi(C)}(1)\right)=$ $h^{0}\left(C, \omega_{C} \otimes \mathcal{N}\right)=q+1$ and $\operatorname{deg}\left(\mathscr{O}_{\psi(C)}(1)\right)=2 q / l$, the well-known fact $\Delta:=$ $1+\operatorname{deg} \mathscr{O}_{\psi(C)}(1)-h^{0}\left(\psi(C), \mathscr{O}_{\psi(C)}(1)\right) \geq 0$ implies that $l=2$ and $\Delta=0$. Hence $\psi(C) \cong \mathbf{P}^{1}$. Thus $C$ is a hyperelliptic curve of genus $q \geq 2$.

Since $E$ is ample and spanned, so is $\pi^{*}(\mathscr{A} \otimes \mathscr{M}) \otimes \mathscr{O}(1)$, and hence $\mathscr{A} \otimes$ $\mathscr{M} \otimes \mathscr{F}$ is ample and spanned. Moreover, $\left.E\right|_{C_{0}} \cong \mathscr{E} \otimes \mathscr{A} \otimes \mathscr{L}$ is ample and spanned, where $C_{0}$ is the minimal section of $\pi$ corresponding to the 1-quotient $\mathscr{F} \rightarrow \mathscr{L}$. Applying (5.5) to $(\mathscr{A} \otimes \mathscr{M}) \otimes \mathscr{F}$ and $\mathscr{E} \otimes(\mathscr{A} \otimes \mathscr{L})$, we have $\alpha+\beta \geq 1$ and $\alpha-e \geq 1$. But, since $c_{2}(E)=2 \alpha+\beta-e=2$, we have

$$
\alpha+\beta=1 \text { and } \alpha-e=1 \text {. }
$$

On the other hand, since $E$ is ample and spanned of rank 2 and since $L$ is spanned, considering the Koszul complex associated with $t \in H^{0}(E)$, we have

$$
4 \leq h^{0}(E) \leq h^{0}(\mathscr{O})+h^{0}\left(\mathscr{I}_{(t)_{0}} \otimes L\right) \leq 1+h^{0}\left(\mathscr{I}_{\left\{P_{1}\right\}} \otimes L\right)=h^{0}(L) .
$$

By using this, we look at the dimension of global sections of $(\mathscr{L} \otimes \mathscr{A})^{\otimes 2} \otimes \mathscr{M}$ and $\mathscr{L} \otimes \mathscr{A} \otimes \mathscr{M}$. Note that $\left.(\mathscr{L} \otimes \mathscr{A})^{\otimes 2} \otimes \mathscr{M} \cong L\right|_{C_{0}}$ and $L\left(-C_{0}\right) \cong \mathscr{O}(1) \otimes$ $\pi^{*}\left(\mathscr{A}^{\otimes 2} \otimes \mathscr{M}\right)$. Since $\mathscr{F}$ is normalized, $h^{0}\left(\mathscr{O}(1) \otimes \pi^{*}\left(\mathscr{A}^{\otimes 2} \otimes \mathscr{M}\right)\right)=h^{0}(C, \mathscr{F} \otimes$ $\left.\left(\mathscr{A}^{\otimes 2} \otimes \mathscr{M}\right)\right)=0$ if $\operatorname{deg}\left(\mathscr{A}^{\otimes 2} \otimes \mathscr{M}\right)=2 \alpha+\beta=\alpha+1<0$, and hence

$$
h^{0}\left((\mathscr{L} \otimes \mathscr{A})^{\otimes 2} \otimes \mathscr{M}\right)=h^{0}\left(\left.L\right|_{C_{0}}\right) \geq h^{0}(L) \geq 4 \text { if } \alpha<-1 .
$$

For $h^{0}(\mathscr{L} \otimes \mathscr{A} \otimes \mathscr{M})$, note that $\mathscr{L} \otimes \mathscr{A} \otimes \mathscr{M}$ fits into the exact sequence

$$
0 \longrightarrow \mathscr{A} \otimes \mathscr{L} \longrightarrow \mathscr{E} \otimes \mathscr{A} \otimes \mathscr{L} \longrightarrow \mathscr{M} \otimes \mathscr{A} \otimes \mathscr{L} \longrightarrow 0,
$$

and that $\left.\mathscr{E} \otimes \mathscr{A} \otimes \mathscr{L} \cong E\right|_{C_{0}}$ and $E\left(-C_{0}\right) \cong \pi^{*}(\mathscr{E} \otimes \mathscr{A})$. Since $\mathscr{E}$ is normalized, $h^{0}\left(E\left(-C_{0}\right)\right)=0$ if $\alpha<0$, hence $h^{0}(\mathscr{E} \otimes \mathscr{A} \otimes \mathscr{L})=h^{0}\left(\left.E\right|_{C_{0}}\right) \geq h^{0}(E) \geq 4$ if $\alpha<0$. On the other hand, by (i), $\operatorname{deg}(\mathscr{A} \otimes \mathscr{L})=\alpha-e=1$, hence $h^{0}(\mathscr{A} \otimes \mathscr{L}) \leq 1$ since $q \neq 0$. Thus we have

(iii) $h^{0}(\mathscr{L} \otimes \mathscr{A} \otimes \mathscr{M}) \geq h^{0}(\mathscr{E} \otimes \mathscr{A} \otimes \mathscr{L})-h^{0}(\mathscr{A} \otimes \mathscr{L}) \geq 3$ if $\alpha<0$.

Now we will deduce a contradiction according to $\alpha$. Since $\mathscr{L} \otimes \mathscr{M} \otimes A$ is ample and spanned, we have $\operatorname{deg}(\mathscr{L} \otimes \mathscr{M} \otimes \mathscr{A})=-e+\beta+\alpha \geq 2$ by $q \neq 0$. So $c_{2}(E)=2 \alpha+\beta-e=2$ implies that $\alpha \leq 0$. But, if $\alpha=0$, then $\beta=-e=1$ by (i). Thus $\operatorname{deg}(\mathscr{A} \otimes \mathscr{L})=1$ and $\operatorname{deg}(\mathscr{L} \otimes \mathscr{A} \otimes \mathscr{M})=2$, so

$$
3 \leq h^{0}(\mathscr{C} \otimes \mathscr{A} \otimes \mathscr{L}) \leq h^{0}(\mathscr{A} \otimes \mathscr{L})+h^{0}(\mathscr{L} \otimes \mathscr{A} \otimes \mathscr{M}) \leq 3,
$$


hence $h^{0}(\mathscr{A} \otimes \mathscr{L})=1$ and $h^{0}(\mathscr{L} \otimes \mathscr{A} \otimes \mathscr{M})=2$. Thus $|\mathscr{A} \otimes \mathscr{L}|=\{x\}$ for some $x \in C$ and $|\mathscr{L} \otimes \mathscr{A} \otimes \mathscr{M}|=g_{2}^{1}$. When $q \geq 3$, since $(\mathscr{A} \otimes \mathscr{L})^{\otimes 2} \otimes \mathscr{M}$ is of degree 3, it is special. Since $(\mathscr{A} \otimes \mathscr{L})^{\otimes 2} \otimes \mathscr{M}$ is spanned, this contradicts (5.6). When $q=2, \operatorname{deg}\left((\mathscr{A} \otimes \mathscr{L})^{\otimes 2} \otimes \mathscr{M}\right)=2 q-1$, hence, by Riemann-Roch, we have $h^{0}\left((\mathscr{A} \otimes \mathscr{L})^{\otimes 2} \otimes \mathscr{M}\right)=2$. On the other hand, $h^{0}\left((\mathscr{A} \otimes \mathscr{L})^{\otimes 2} \otimes \mathscr{M} \otimes \mathscr{O}(-x)\right)=$ $h^{0}(\mathscr{L} \otimes \mathscr{A} \otimes \mathscr{M})=2$. This implies that $x$ is a base point of $(\mathscr{A} \otimes \mathscr{L})^{\otimes 2} \otimes \mathscr{M}$, contradiction. Thus $\alpha \leq-1$.

Then we claim that

$$
\alpha+\beta-e \equiv 0 \quad(\bmod 2) .
$$

Since $\mathscr{L} \otimes \mathscr{M} \otimes \mathscr{A}$ is ample and spanned of degree $\alpha+\beta-e$, by (5.6), we have only to show that $\mathscr{L} \otimes \mathscr{M} \otimes \mathscr{A}$ is special. Since $\alpha \leq-1$, by (iii), we have $h^{0}(\mathscr{L} \otimes \mathscr{M} \otimes \mathscr{A}) \geq 3$. Moreover, by $\beta \leq q$ (see [27, Theorem 1]) and by (i), $\operatorname{deg}(\mathscr{L} \otimes \mathscr{M} \otimes \mathscr{A})=\alpha+\beta-e=1+\beta \leq 1+q$. Hence, by Riemann-Roch, $h^{1}(\mathscr{L} \otimes \mathscr{M} \otimes \mathscr{A}) \geq 1$. Thus $\mathscr{L} \otimes \mathscr{M} \otimes \mathscr{A}$ is special, as required.

When $\alpha=-1, \beta=-e=2$ by (i). This contradicts (iv). Hence $\alpha \leq-2$.

Finally we claim that

$$
2 \alpha-2 e+\beta \equiv 0 \quad(\bmod 2) .
$$

As above, since $\left.L\right|_{C_{0}} \cong(\mathscr{L} \otimes \mathscr{A})^{\otimes 2} \otimes \mathscr{M}$ is ample and spanned of degree $2 \alpha-2 e+\beta$, by (5.6), we have only to show that $(\mathscr{L} \otimes \mathscr{A})^{\otimes 2} \otimes \mathscr{M}$ is special. Since $\alpha \leq-2$, by (ii), we have $h^{0}\left((\mathscr{L} \otimes \mathscr{A})^{\otimes 2} \otimes \mathscr{M}\right) \geq 4$. By $\beta \leq q$ and (i), $\operatorname{deg}\left((\mathscr{L} \otimes \mathscr{A})^{\otimes 2} \otimes \mathscr{M}\right)=2 \alpha-2 e+\beta=2+\beta \leq 2+q$. Hence, by Riemann-Roch, $h^{1}\left((\mathscr{L} \otimes \mathscr{A})^{\otimes 2} \otimes \mathscr{M}\right) \geq 1$. Thus $(\mathscr{L} \otimes \mathscr{A})^{\otimes 2} \otimes \mathscr{M}$ is special, as required.

Now we get to the final contradiction; (iv) and (v) imply that $\alpha-e \equiv 0$ (mod 2), which contradicts (i). Thus $q \leq 1$.

Since $s(E) \geq \operatorname{dim} X=2$, we have $c_{2}(E) \geq 2$. So we have $q(X)=0$ or 1 .

Step 5.7. Assume that $X$ is an eth Hirzebruch surface $\mathbf{F}_{e}$ with the projection $\pi: X \rightarrow \mathbf{P}^{1}$, the minimal section $h$ and with a fibre $f$. Assume that $K \otimes L$ is not ample. Then $s(E)=2 c_{2}(E)-1$ if and only if $(X, E)$ is isomorphic to one of (12).

Proof. If $s(E)=2 c_{2}(E)-1$, it follows from (5.1) that the bundle $E$ is of the form $\mathscr{O}(h+a f) \oplus \mathscr{O}(h+b f)$. Then $E$ is ample and spanned if and only if $a, b>e$. If $a=b$, then $s(E)=2 c_{2}(E)$ by (2.2). Thus we may assume that $b>a>e$. Then $s(E)=3 a+b-2 e+1$ by $(2.2)$ and $c_{2}(E)=-e+a+b$, hence $s(E)=2 c_{2}(E)-1$ if and only if $b=a+2$, as required.

Finally we determine the bundles $E$ with $s(E)=2 c_{2}(E)-1$, on an elliptic geometrically ruled surface $X$. Before doing this, we note the following lemmas about a normalized and indecomposable rank-2 vector bundle on an elliptic curve.

Lemma 5.8. Let $\mathscr{E}$ be an indecomposable and normalized vector bundle of rank 2 on an elliptic curve $C$.

(1) If $\operatorname{deg} \mathscr{E}=0$, then $h^{0}(\mathscr{E})=1, h^{1}(\mathscr{E})=1$, and $h^{0}(\mathscr{E} \otimes \mathscr{E} \vee)=2$.

(2) If $\operatorname{deg} \mathscr{E}=1$, then $h^{0}(\mathscr{E})=1, h^{1}(\mathscr{E})=0$, and $h^{0}(\mathscr{E} \otimes \mathscr{E} \vee)=1$. 
Proof. This easily follows from [18, p. 337, (2.15)] and (2.3)(2) (see also [6, Lemma 1] for (2)).

Lemma 5.9. Let $\mathscr{E}$ and $C$ be as in (5.8). Assume that $\operatorname{deg} \mathscr{E}=1$. Let $\mathscr{N}$ be a line bundle of degree 1 on $C$. Then, for each $x \in C$, there exists a line bundle $\mathscr{C}^{x}$ of degree 1 on $C$ such that

$$
0 \longrightarrow \mathscr{O}_{C}(x) \longrightarrow \mathscr{E} \otimes \mathscr{N} \longrightarrow \mathscr{C}^{x} \otimes \mathscr{O}_{C}(x) \longrightarrow 0
$$

is exact. Therefore, $\mathscr{E} \otimes \mathcal{N}$ is generated by its global sections.

Proof. See, for example, [6, p. 229].

Step 5.10. Assume that $X$ is a geometrically ruled surface over an elliptic curve $C$ with the projection $\pi: X \rightarrow C$ and that $K \otimes L$ is not ample. Then $s(E)=$ $2 c_{2}(E)-1$ if and only if $(X, E)$ is isomorphic to one of (13).

Proof. If $s(E)=2 c_{2}(E)-1$, by (5.1), we have $X=\mathbf{P}_{C}(\mathscr{F})$ and $E=$ $\pi^{*}(\mathscr{E} \otimes \mathscr{A}) \otimes \mathscr{O}(1)$, where $\mathscr{F}, \mathscr{E}, \mathscr{A}$, and $\mathscr{O}(1)$ are as in $(5.1)$. Let $C_{0}$ be the member of $|\mathscr{O}(1)|$ corresponding to the quotient $\mathscr{F} \rightarrow \mathscr{L}$. First we claim that $E$ is indecomposable. To the contrary, we assume that $E=M_{1} \oplus M_{2}$ for line bundles $M_{i}(i=1,2)$. By $(2.2)$, a smooth and irreducible member $D_{1} \in\left|M_{1}\right|$ or $D_{2} \in\left|M_{2}\right|$ is of $\Delta$-genus zero, hence a smooth rational curve, so $g\left(X, M_{1}\right)=0$ or $g\left(X, M_{2}\right)=0$. Therefore $X$ must be rational (see [10, (12.1), (5.10)]), contradiction. Hence $E$ is indecomposable and so is $\mathscr{E}$. In particular, the exact sequences (i) and (iii) in (5.1) are nonsplit and therefore, for $\mathscr{M}$ in (i), there are two possibilities (see [18, p. 377, (2.15)]):

Case I. $\mathscr{M} \cong \mathscr{O}_{C}$, hence $\operatorname{deg} \mathscr{E}=0$.

Case II. $\mathscr{M} \cong \mathscr{O}_{C}(x)$ for some $x \in C$, hence $\operatorname{deg} \mathscr{E}=1$.

Now we show that Case I does not occur. To exclude Case I, we calculate $s(E)$ by using the exact sequence (iii) in (5.1). Clearly $h^{0}\left(E^{\vee}\right)=h^{1}\left(E^{\vee}\right)=0$. By (2.5)(2), $\left.\operatorname{deg} E\right|_{C_{0}}=c_{1}(E) \cdot C_{0}=-2 e+2 \alpha \geq 3$, hence $\alpha-e \geq 2$ and $\alpha \geq 2+e \geq 1$. Therefore

$$
0=h^{1}(\mathscr{A})+h^{1}(\mathscr{L} \otimes \mathscr{A}) \geq h^{1}(\mathscr{F} \otimes \mathscr{A})=h^{1}\left(\pi^{*} \mathscr{A} \otimes \mathscr{O}(1)\right) .
$$

Consequently, $h^{0}(E)=2 h^{0}\left(\pi^{*} \mathscr{A} \otimes \mathscr{O}(1)\right)$. By (2.2) and (5.8),

$$
\begin{aligned}
s(E) & =h^{0}(E)-h^{0}\left(E \otimes E^{\vee}\right)=h^{0}(E)-h^{0}\left(\mathscr{E} \otimes \mathscr{E}^{\vee}\right) \\
& =2\left(h^{0}\left(\pi^{*} \mathscr{A} \otimes \mathscr{O}(1)\right)-1\right) \not \equiv 2 c_{2}(E)-1 \quad(\bmod 2),
\end{aligned}
$$

contradiction (see also Remark 5.11).

In Case II, the exact sequence (iii) in (5.1) is

$$
0 \longrightarrow \pi^{*} \mathscr{A} \otimes \mathscr{O}(1) \longrightarrow E \longrightarrow \pi^{*} \mathscr{A}(x) \otimes \mathscr{O}(1) \longrightarrow 0 .
$$

Since $\left.E\right|_{C_{0}}$ is ample and spanned, $\left.\operatorname{deg} E\right|_{C_{0}}=c_{1}(E) \cdot C_{0}=-2 e+2 \alpha+1 \geq 3$ by (2.5), hence $\alpha-e \geq 1$, as required.

Conversely, we show that every vector bundle $E$ in (13) is ample and spanned with $s(E)=2 c_{2}(E)-1$. Note that $E$ fits into the exact sequence (i). One checks easily that, for every line bundle $\mathscr{Z}$ of $\operatorname{deg} \mathscr{Z}=0$ on $C$, setting $\mathscr{A}^{\prime}=\mathscr{A} \otimes \mathscr{Z}$, we have

$$
\begin{aligned}
h^{0}\left(\pi^{*} \mathscr{A}^{\prime} \otimes \mathscr{O}(1)\right) & =h^{0}\left(\mathscr{F} \otimes \mathscr{A}^{\prime}\right)=2 \alpha-e, \\
h^{0}\left(\pi^{*} \mathscr{A}^{\prime}(x) \otimes \mathscr{O}(1)\right) & =h^{0}\left(\mathscr{F} \otimes \mathscr{A}^{\prime}(x)\right)=2 \alpha-e+2, \\
h^{1}\left(\pi^{*} \mathscr{A}^{\prime} \otimes \mathscr{O}(1)\right) & =h^{1}\left(\mathscr{F} \otimes \mathscr{A}^{\prime}\right)=0 .
\end{aligned}
$$


Consequently, (i) is globally exact and $h^{0}(E)=4 \alpha-2 e+2$. It follows from this and (2.2) and (5.8) that

$$
s(E)=h^{0}(E)-h^{0}\left(E \otimes E^{\vee}\right)=4 \alpha-2 e+2-h^{0}\left(\mathscr{E} \otimes \mathscr{E}^{\vee}\right)=2 c_{2}(E)-1,
$$

if $E$ is ample and spanned. By [18, p. 382, (2.20), (2.21); p. 385, Example 2.12], the global exactness of (i) implies that $E$ is ample and spanned if $\alpha-e \geq 2$ and that $E$ is ample if $\alpha-e=1$. It remains to show that $E$ is spanned when $\alpha-e=1$. (In the case $e=-1$, the proof is in [6].) Since (i) is globally exact and since $\pi^{*} \mathscr{A}(x) \otimes \mathscr{O}(1)$ is spanned, $H^{0}(E)$ spans $E$ at each point of $X$ away from $\mathrm{Bs}\left|\pi^{*} \mathscr{A} \otimes \mathscr{O}(1)\right|$.

When $e=-1, \alpha=0$ and $\mathscr{F} \otimes \mathscr{A}$ is normalized, there exists a section $D \in\left|\pi^{*} \mathscr{A} \otimes \mathscr{O}(1)\right|$ of $\pi$ with $D^{2}=1$. Since $\left.\left.\left.E\right|_{D} \cong \pi^{*} \mathscr{E}\right|_{D} \otimes \mathscr{O}(D)\right|_{D}$ is spanned by $(5.9)$ and since $h^{1}(E \otimes \mathscr{O}(-D))=h^{1}(\mathscr{E})=0$ by $(5.8), H^{0}(E)$ also spans $E$ at each point of $D$, as required.

When $e=0$ and $\mathscr{F}$ is indecomposable, $\mathscr{A}=\mathscr{O}_{C}(z)$ for some $z \in C$ and hence Bs $\left|\mathscr{O}(1) \otimes \pi^{*} \mathscr{A}\right| \subseteq \operatorname{Supp}\left(C_{0}+\pi^{*} z\right)$. By (5.9), for each $z^{\prime} \neq z \in C, E$ fits into another exact sequence $0 \rightarrow \pi^{*} \mathscr{O}_{C}\left(z^{\prime}\right) \otimes \mathscr{O}(1) \rightarrow E \rightarrow \pi^{*} \mathscr{B} \otimes \mathscr{O}(1) \rightarrow 0$, where $\mathscr{B}$ is a line bundle of degree 2 over $C$. Thus $H^{0}(E)$ spans $E$ at each point away from $C_{0}$. Since $h^{1}\left(E \otimes \mathscr{O}\left(-C_{0}\right)\right)=0$, and since $\left.E\right|_{C_{0}} \cong$ $\mathscr{E} \otimes \mathscr{A} \otimes \mathscr{O}_{C_{0}}\left(C_{0}\right)$ is spanned by (5.9), $E$ is spanned.

When $e \geq 0$ and $\mathscr{F}$ is decomposable, $\mathscr{F}=\mathscr{O}_{C} \oplus \mathscr{L}, \operatorname{deg} \mathscr{L}=-e \leq 0$ and $\mathscr{A} \otimes \mathscr{L}=\mathscr{O}_{C}(z)$ for some $z \in C$. Let $D \in\left|\mathscr{O}(1) \otimes \pi^{*}\left(\mathscr{L}^{-1}\right)\right|$ be a section of $\pi$ corresponding to $\mathscr{O}_{C} \oplus \mathscr{L} \rightarrow \mathscr{O}_{C}$. Then Bs $\left|\mathscr{O}(1) \otimes \pi^{*}(\mathscr{A})\right| \subseteq D+\pi^{*} z$. As above, $H^{0}(E)$ spans $E$ at each point away from $\operatorname{Supp}(D)$. Since $h^{1}(E(-D))=$ $h^{1}(\mathscr{E} \otimes \mathscr{A} \otimes \mathscr{L})=0$ and since $\left.E\right|_{D} \cong \mathscr{E} \otimes \mathscr{O}_{D}(1) \otimes \mathscr{A}, E$ is spanned. This completes the proof.

Remark 5.11. Let $(X, E)$ be the pair as in Case $I$ in (5.10). Then $E$ is ample and spanned if and only if $\alpha-e \geq 2$. In this case, $c_{2}(E)=2 \alpha-e$, and $s(E)=2 c_{2}(E)-2$ hold. Thus the $(X, E)$ 's are counterexamples to (6.1) in [4]. Consequently, the main theorem (0.1) of [5] is false. On the other hand, one checks immediately that $s(E)=6=2 c_{2}(E)-2$ for $(X, E) \cong$ $\left(\mathbf{P}^{2}, \mathscr{O}(1) \oplus \mathscr{O}(4)\right)$. Hence $(X, E)$ satisfies the conditions in (0.1) [5], but does not appear in the list in (0.1) of [5], either.

\section{AN APPLICATION: AMPLE AND SPANNED RANK-2 BUNDLES OF $c_{2}=2$ ON SURFACES}

In this section, as an application of (3.1) and (4.1) together with a result of Ballico [3, (4.2)], we classify rank-2 ample and spanned vector bundles on smooth complex projective surfaces with $c_{2}(E)=2$.

Theorem 6.1. Let $E$ be an ample and spanned rank-2 vector bundle on a smooth projective surface $X$. Then $c_{2}(E)=2$ if and only if $(X, E)$ is one of the following:

(1) $(X, E) \cong\left(\mathbf{P}^{2}, \mathscr{O}(1) \oplus \mathscr{O}(2)\right)$.

(2) $(X, E) \cong\left(\mathbf{Q}^{2}, \mathscr{O}(1)^{\oplus 2}\right)$, where $\mathbf{Q}^{2}$ is a smooth quadric in $\mathbf{P}^{3}$.

(3) $X$ is isomorphic to a geometrically ruled surface $\mathbf{P}_{C}(\mathscr{F})$ over an elliptic curve $C$ with the projection $\pi: \mathbf{P}_{C}(\mathscr{F}) \rightarrow C$ and with the tautological line bundle $\mathscr{O}(1)$, and $E \cong \pi^{*}(\mathscr{E}) \otimes \mathscr{O}(1)$. Here $\mathscr{F}$ and $\mathscr{E}$ are indecomposable rank-2 vector bundles on $C$ of degree 1 . 
(4) There exists a finite morphism $f: X \rightarrow \mathbf{P}^{2}$ of degree 2 and $E \cong$ $f^{*}\left(\mathscr{O}(1)^{\oplus 2}\right)$.

Proof. If $E$ is ample and spanned with $c_{2}(E)=2$, then, by the definition of $s(E), 2=\operatorname{dim} X \leq s(E) \leq 2 c_{2}(E)=\operatorname{dim} X \cdot c_{2}(E)$. When $s(E)=4$, by (3.1), $(X, E)$ is isomorphic to one of $(1)$ or $(2)$. When $s(E)=3$, by $(4.1),(X, E)$ is isomorphic to one of (3). When $s(E)=2$, by [3, (4.2)], $(X, E)$ is isomorphic to one of (4). The converse is obvious.

\section{REFERENCES}

1. E. Arbarello, M. Cornalba, P.A. Griffiths, and J. Harris, Geometry of algebraic curves, vol. I, Grundlehren Math. Wiss., Bd. 267, Springer-Verlag, New York and Berlin, 1984.

2. M. Artin, On deformation of singularities, Tata Inst. Fund. Res. Lectures on Math. and Phys., vol. 54, Tata Inst. Fund. Res., Bombay, 1976.

3. E. Ballico, Rank-2 vector bundles with many sections and low $c_{2}$ on a surface, Geom. Dedicata 29 (1989), 109-124.

4. J. 38 (1989), 471-487.

5. __ Construction of bundles on surfaces with low $c_{2}$, Indiana Univ. Math. J. 38 (1989), 489-496.

6. E. Ballico and A. Lanteri, An indecomposable rank- 2 vector bundle the complete linear system of whose determinant consists of hyperelliptic curves, Boll. Un. Mat. Ital. A (7) (1989), 225-230.

7. _ Ample and spanned rank- 2 vector bundles with $c_{2}=2$ on complex surfaces, Arch. Math. (Basel) 56 (1991), 611-615.

8. A. Biancofiore, M. L. Fania, and A. Lanteri, Polarized surfaces with hyperelliptic sections, Pacific J. Math. 143 (1990), 9-24.

9. S. Bloch and D. Gieseker, The positivity of the Chern classes of an ample vector bundle, Invent. Math. 12 (1971), 112-117.

10. T. Fujita, Classification theories of polarized varieties, London Math. Soc. Lecture Note Ser., vol. 155, Cambridge Univ. Press, London and New York, 1990.

11. __ On adjoint bundles of ample vector bundles, Complex Algebraic Varieties (Proc., Bayreuth 1990) (K. Hulek et al., eds.), Lecture Notes in Math., vol. 1507, Springer-Verlag, Berlin and New York, 1992, pp. 105-112.

12. $\frac{}{1-16}$, Ample vector bundles of small $c_{1}$-sectional genera, J. Math. Kyoto Univ. 29 (1989),

13. W. Fulton, Intersection theory, Ergeb. Math. Grenzgeb., Bd. 2, Springer-Verlag, Berlin and New York, 1988.

14. D. Gieseker, p-ample bundles and their Chern classes, Nagoya Math. J. 43 (1971), 91-116.

15. P. Griffiths and J. Harris, Residues and zero-cycles on algebraic varieties, Ann. of Math. (2) 108 (1978), 461-505.

16. A. Grothendieck, Eléments de géométrie algébrique. II, IV-3, Inst. Hautes Études Sci. Publ. Math. 8 (1961), 28 (1966).

17. _ Techniques de construction et théorèmes d'existence en géométrie algébrique: Les schémas de Hilbert, Sém. Bourbaki, exposé 221 Secrétariat Math., Paris.

18. R. Hartshorne, Algebraic geometry, Graduate Texts in Math., vol. 52, Springer-Verlag, Berlin and New York, 1977.

19. D. Laksov, Indecomposability of restricted tangent bundles, Young tableaux and Schur functors in algebra and geometry, Astérisque 87-88 (1981), 207-220.

20. A. Lanteri, Variazioni sul tema classico delle sezioni iperellittiche, Rend. Sem. Mat. Fis. Milano 57 (1987), 533-547. 
21. __ A note on k-dimensional double solids, Rend. Sem. Mat. Brescia 10 (1988), 1-9.

22. A. Lanteri and H. Maeda, Adjoint bundles of ample and spanned vector bundles on algebraic surfaces, J. Reine Angew. Math. J. Reine Angew. Math. 433 (1992), 181-199.

23. R. Lazarsfeld, Some application of the theory of positive vector bundles, Complete Intersections, Lecture Notes in Math., vol. 1092, Springer-Verlag, Berlin and New York, 1984, pp. 29-61.

24. T. Matsubara, Ample rank-2 bundles on a surface with sections whose zero loci spread widely, preprint.

25. H. Matsumura, Commutative ring theory, Cambridge Stud. Adv. Math., vol. 8, Cambridge Univ. Press, London and New York, 1986.

26. D. Mumford, Lectures on curves on algebraic surfaces, Ann. of Math. Stud., vol. 59, Princeton Univ. Press, Princeton, NJ, 1966.

27. M. Nagata, On self-intersection number of a section on a ruled surface, Nagoya Math. J. 37 (1970), 191-196.

28. C. Okonek, M. Schneider, and H. Spindler, Vector bundles on complex projective spaces, Progr. Math., vol. 3, Birkhäuser, Boston, 1980.

Department of Mathematics, School of Science and Engineering, Waseda University, 3-4-1 OhKubo, ShinjuKu-Ku, Tokyo, 169, JaPAN

E-mail address: noma@cfi.waseda.ac.jp 